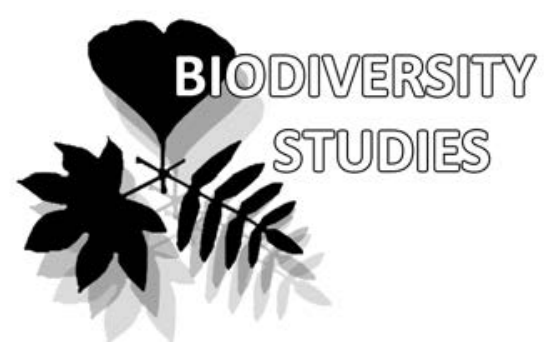

\title{
The moss flora of the Badzhal Mountain Range (Khabarovsk Territory, Russian Far East)
}

\author{
Olga Yu. Pisarenko ${ }^{1 *}$, Vladimir E. Fedosov ${ }^{2,3}$, \\ Kirill A. Korznikov ${ }^{3}$, Anna V. Shkurko ${ }^{4}$, Elena A. Ignatova ${ }^{2}$
}

Olga Yu. Pisarenko ${ }^{1 *}$

e-mail: o_pisarenko@mail.ru

Vladimir E. Fedosove,

e-mail: fedosov_v@mail.ru

Kirill A. Korznikov ${ }^{3}$

e-mail: korzkir@mail.ru

Anna V. Shkurko ${ }^{4}$

e-mail: shen-ku@bk.ru

Elena A. Ignatova ${ }^{2}$

e-mail: arctoa@list.ru

${ }^{1}$ Central Siberian Botanical Garden

SB RAS, Novosibirsk, Russia

${ }^{2}$ Lomonosov Moscow State

University, Faculty of Biology, Geobotany

Dept., Moscow, Russia

${ }^{3}$ Botanical Garden-Institute FEB RAS, Vladivostok, Russia

${ }^{4}$ Tsitsin Main botanical Garden RAS, Moscow, Russia

\section{* corresponding author}

Manuscript received: 01.12.2021

Review completed: 24.02 .2022

Accepted for publication: 02.03 .2022

Published online: 04.03.2022

\begin{abstract}
A B S T R A C T
On the southern periphery of Badzhal Mountain Range, an area of about 25 sq. $\mathrm{km}$, we found 313 species of mosses, listed here with distribution along with the altitudinal belts, ecotopes, and substrates. Among them, 73 species are newly recorded for the middle part of Khabarovsk Territory, and 39 of them are new to the whole Khabarovsk Territory, including rare species with no or few previously known records in Russia such as Campylopus gracilis, Sematophyllum substrumulosum, Anomodon solovjovii, Bryoerythrophyllum chenii, Dicranum setifolium, Ditrichum macrorbynchum, Haplobymenium longinerve, Okamurea bakkoniensis, Orthotrichum rogeri, Sphagnum miyabeanum, S. subnitens; identity of several species needs in specially focused taxonomic studies. Studied flora is compared with ones of Zeysky State Nature Reserve, Upper Bureya, and a combined list of mosses of Botchinsky Nature Reserve and Tordoki-Yani Mt. Among all these floras, the proportion of Eastern species in Badzhal is the highest. Main types of vegetation and bryophyte ecotopes are briefly characterized; distribution of species along an altitudinal gradient among considered types of habitats and substrates is discussed.
\end{abstract}

Keywords: mosses, flora, biodiversity, rare species, phytogeography

\section{P E 3 Ю M E}

Писаренко О.Ю., Федосов В.Э., Корзников К.А., Шкурко А.В., Игнатова Е.А. К бриофморе БаАжамьского хребта (Хабаровский край, Российский Аамьний Восток). Проведено бриологическое обслеАование ключевого участка южного макросклона БаАжальского хребта; на территории площадью около 25 кв. км выяв ено 313 видов мхов. Впервые в средней части Хабаровского края зАесь выявлены 73 вила мхов, из них 39 виАов - новые Аля всего Хабаровского края. Наиболее интересны находки Campylopus gracilis, Sematophyllum substrumulosum, Anomodon solovjovii, Bryoerythrophyllum chenii, Dicranum setifolium, Ditrichum macrorbynchum, Haplohymenium longinerve, Okamurea bakkoniensis, Orthotrichum rogeri, Sphagnum miyabeanum, S. subnitens, ранее не известных с территории России или известных из немногих местонахожАений. Аля ряда находок родство и статус требует специальных таксономических исследований. Приводится аннотированный список с указанием занимаемых местообитаний и субсстратов и распространения на высотном профиле. Аана характеристика основных типов растительных сообществ и местообитаний мхов, эколого-ценотическое и высотное распределение вилов мхов описано. Исследованная флора сопоставлена с флорами Зейского заповедника, Верховий Буреи и объединенной флоры Ботчинского заповедника и г. Тордоки-Яни. В ряду сравниваемых бриофлор исследованная территория вылеляется повышенным участием восточных вилов.

Ключевые слова: мхи, флора, биоразнообразие, редкие виды, фитогеография
The southern half of the Russian Far East is exciting since Holarctic boreal flora is gradually replaced here by East Asian one. Moreover, moss flora of the upper course of the Bureya River was found to include several rare Asian taxa such as Actinothuidium hookeri (Mitt.) Broth and Cryphaea amurensis Ignatov. So, further study of mountain ranges in the middle part of Khabarovsk Territory may improve our knowledge and understanding of their distribution and yield other interesting records. Our attention was focused on Badzhal Mountain Range. The territory is quite well studied in the diversity of vascular plants (Shlotgauer 2015). The analysis of vascular flora demonstrated its remarkable peculiarity (Shlotgauer 2014, 2015). In particular, it was emphasized that the geographical peculiarity of the Bad- zhal range is its position in the zone of the increadible convergence of the ultracontinental and marine climates, which determines the co-existence of floristic complexes sharply contrasting in ecology and origin. This feature proves the attractiveness of the territory for further botanical exploration. As for the mosses, before our research, the Badzhal ridge was a bryological blank spot; only 49 species were listed for its southwest periphery on the boundary with the Amur Province (Cherdantseva et al. 1997).

In 2016, Botanical Garden-Institute of Far Eastern Branch of the Russian Academy of Sciences organized an expedition to study flora and vegetation of key plots on the Southern macroslope of Badzhal mountains, in the upper reaches of the Yarap River (Figs 1, 2). Already at the 

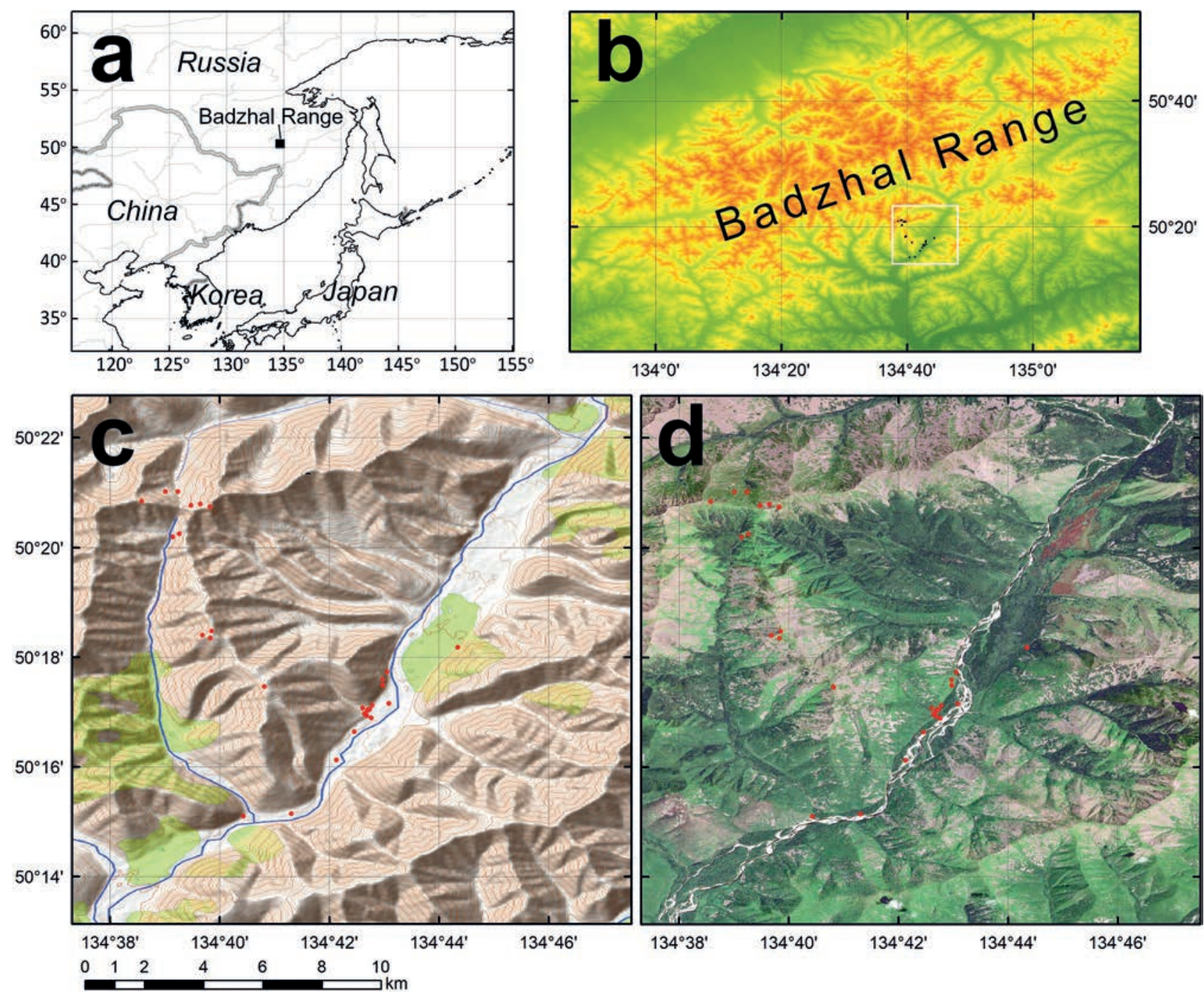

Figure 1 Collecting localities in the Badzhal Range; a: geographic position of the study area ; b: physical map of the Badzhal Range; c: topographic map of the study area; $d$. satellite image of the study area

beginning of the work, many new and interesting moss species were revealed; some of the most exciting findings have already been published (Pisarenko \& Fedosov 2016, Ellis et al. 2017, Pisarenko et al. 2017, Ellis et al. 2018, Cherdantseva et al. 2018). The present paper summarizes the results of the bryological survey of that site.

\section{STUDY AREA AND OBSERVATION RESULTS}

About 1000 samples collected on the key plot were treated and placed in MW and NSK; full labels are incorporated into the DataBase Arctoa (Ivanov et al. 2017, Herbarium... 2020). Taxa new for the middle part of Khabarovsk Territory ("Khm" according to scheme of delimitation accepted in Moss flora of Russia, http://arctoa.ru/ Flora/regions.php) are marked with an asterisk.

In the check-list below compiled based on these collections (Table 1), nomenclature follows "Mosses of the southern Russian Far East" (Cherdantseva et al. 2018) with recent improvements; in the last case, synonyms are given. Species are annotated by the altitudinal range. Frequency is given by three altitude belts (forest belt, dwarf thickets belt, alpine tundra belt); it is ranked as common-sporadic-rareunique (c-s-r-u). Also, the table shows the occurrence of species in the main types of habitats (as described above), as well as species composition for all the types.

The study area is located in the upper part of the Yarap River Basin. On the key plot, the altitude varies from about $500 \mathrm{~m}$ above sea level in the floodplain of the Yarap River to about $1500-2000 \mathrm{~m}$, approaching the watershed. A relief is deeply dissected, with narrow valleys of mountain rivers and steep slopes. Bedrocks are composed of Cretaceous felsic rocks, mainly lavas, less often tuffs. Permian siliceous shales, conglomerates, clay shales, siltstones, and sandstones occur in some areas. Quaternary formations are represented by alluvial deposits in valleys and diluvial-colluvial block-gravel deposits with gruss and loam on mountain slopes. Pleistocene glacial deposits associated with glacial landforms occur in the upper parts of mountains slopes and tops.

The climate is monsoon, with wet, relatively warm summer and dry cold winter. The nearest weather station Chegdomyn is situated $130 \mathrm{~km}$ northwest. Here the mean annual temperature is $-2^{\circ} \mathrm{C}$ (July $20.3^{\circ} \mathrm{C}$, January $-29.7^{\circ} \mathrm{C}$ ), the mean annual precipitation is $675 \mathrm{~mm}(592 \mathrm{~mm}$ from April to September), however, according to extrapolation by Petrov et al. (2000) in montane areas of the Badzhal Range annual precipitations reach above 1000 with $70 \%$ concen- 

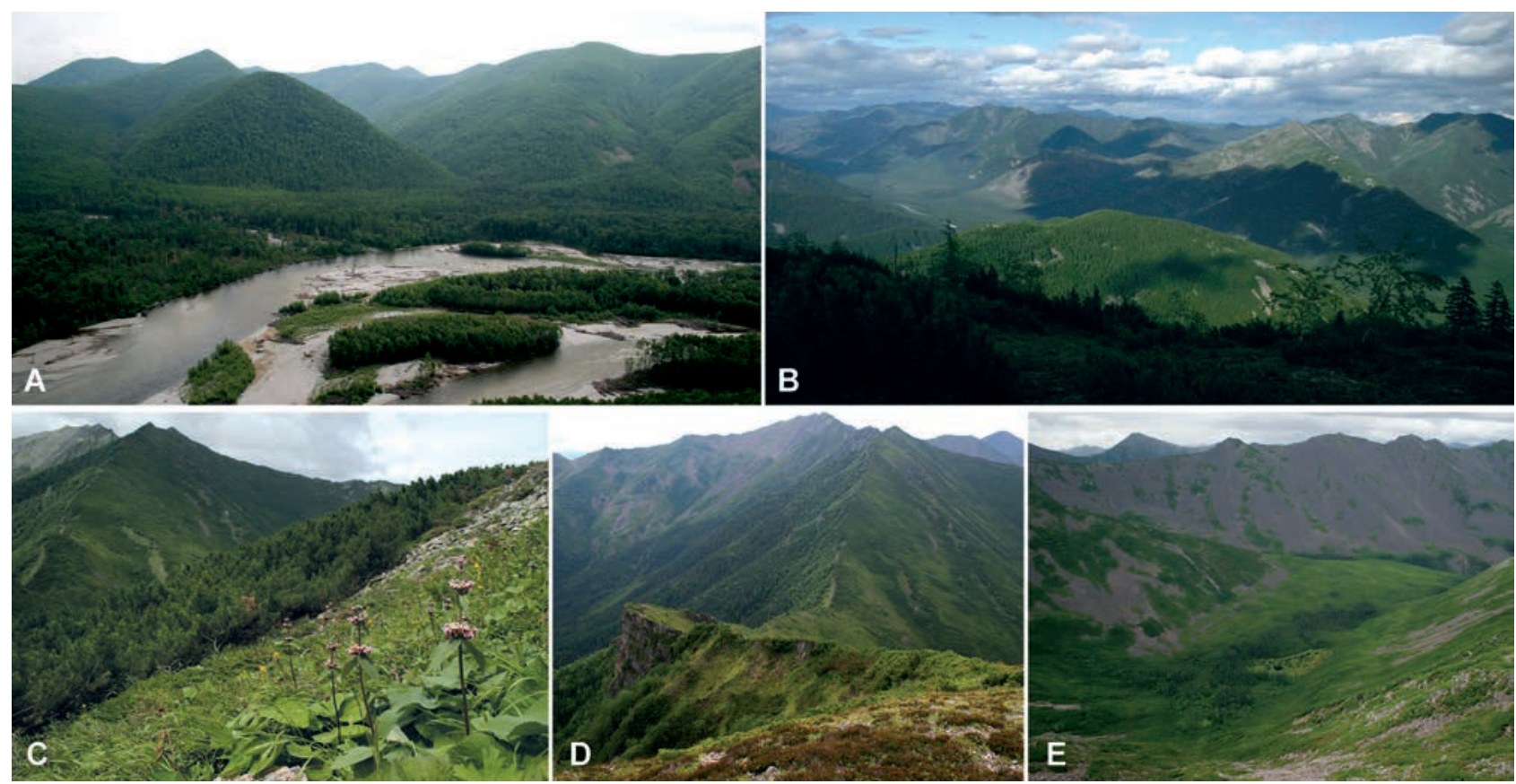

Figure 2 Landscape photographs: A - Yarap River, forested slopes; B - watersheds view; C, D - steep slopes above the forest line: mosaic of dwarf pine thickets with mountain tundra and alpine herbaceous communities; E - screes occupy vast areas, swamps are very rare (a small massif is visible at the foot of the slope). Photos by Pisarenko, 2016

trated in summertime. Thus, according to the bioclimate type of the area is southern boreal-oroboreal maritime (Nakamura et al. 2007).

\section{VEGETATION OF THE STUDY SITE}

Vegetation of the area is considered in a number of studies (Sochava 1934, Shlotgauer 1990, Osipov 2002, and others); in the review below, they are supplemented by our field studies and observations.

The following altitudinal belts are distinguished in the vegetation of the Badzhal Mountain Range: alpine tundra, subalpine dwarf pine and dwarf alder thickets, boreal larch and boreal spruce forests, deciduous forests (Krestov 2003). The latter occurs only at the foothills of the ridge up to $300 \mathrm{~m}$ altitudes, outside the territory we have surveyed. The heights of the boundaries of vegetation belts depend on the exposure and the steepness of the slopes (Krestov 2003); the absolute value varies up to $200 \mathrm{~m}$. Boreal forest belt occupy the largest areas in the studied territory; boreal forests alternated with screes cover mountain slopes from valley bottom up to $1300-1500 \mathrm{~m}$ a.s.l. From the altitudes $1300-1500 \mathrm{~m}$ subalpine belt starts; its vegetation is composed of dwarf pine and dwarf alder thickets; these communities dominate up to about $1600 \mathrm{~m}$. Alpine tundra-like communities and meadows occur from $1500 \mathrm{~m}$, becoming common from $1700 \mathrm{~m}$.

Below the main types of plant communities are considered, emphasizing on the most important for mosses. For all vegetation types, the entire species composition of mosses is given in Table 1. Names of vascular plants follow The Plant List database (http://www.theplantlist.org/), except Larix cajanderi Mayr (since in the database, the species is given as a synonym of $L$. gmelinii).

\section{Valley dark conifer forests}

Valley dark conifer forests are dominated by Picea jezoensis and Abies nephrolepis but also contains small amounts of Betula lanata, B. platyphylla, Larix cajanderi, Pinus koraiensis, Populus suaveolens, and Sorbus sibirica. A tree layer usually consists of 2 or 3 sublayers with mean heights 25,15 and $10 \mathrm{~m}$. A mean canopy cover is $70-80 \%$. A shrub layer is 150-200 cm tall, the total cover is 20-30\%, composed of Lonicera maximowiczii, Prunus padus, Ribes mandshuricum, Rosa acicularis, Sorbaria sorbifolia, and Swida alba. A herb layer mean cover is $40 \%$, composed by Aruncus dioicus, Atbyrium filix-femina, Calamagrostis langsdorffii, Carex pallida, Carex falcata, Circea alpina, Diplazium sibiricum, Leptorumohra amurensis, Mitella nuda, Oxalis acetosella, Parasenecio hastatus, Phegopteris connectilis, Saussurea triangulata, Smilacina davurica.

A moss layer is well developed; it covers up to $90 \%$ in the patches where the herb layer is sparse and represented by small boreal species as Maianthemum bifolium, Oxalis acetosella, Trientalis europaea, etc. Moss composition varies depending on local environments; Hylocomiadelphus triquetrus, Hylocomium splendens, Pleurozium scbreberi, Dicranum spp. are the most common (Table 1, 1). However, in typical hemiboreal forests, which cover bottom of Yarap River valley, the polydominant moss layer along with boreal species comprises Atrichum flavisetum, Plagiomnium spp., Brachythecium spp., Sciuro-hypnum spp., Rhodobryum roseum, Thuidium spp., Gollania ruginosa, Hypnum cupressiforme ssp. subjulaceum, etc. (Table 1,1). These communities are characterized by relatively constant conditions of temperature and humidity. They are the richest in diversity and abundance of mosses. In particular, the presence of old-aged poplar trees contributes to the diversity of epiphytes, housing such species as Anacamptodon latidens, Brachythecium buchananii, Bryoerythrophyllum chenii, 
Table 1. Species list of the key plot of the Badzhal range.

Frequency is shown by altitude belts $(\mathbf{F}-$ forest belt, $\mathbf{D}$ - dwarf thickets belt, $\mathbf{T}$ - tundra belt); it is ranked as commonsporadic-rare-unique $(\mathbf{c}-\mathbf{s}-\mathbf{r}-\mathbf{u})$.

Types of habitats (described in the text): 1 - Valley dark conifer forests; 2 - Mountain dark conifer forests; 3 - Mountain larch forests; 4 - wetlands, all together; 5 - dwarf pine and dwarf alder thickets; 6 - Mountain tundra; 7 - Alpine herbaceous communities; 8 - snowbeds; 9 - watercourse banks and beds; 10 - cliffs; 11 - rock fields.

The types of substrates are abbreviated as follow: $\mathbf{b}$ - bark on tree stands above grass layer, dw - decaying wood, eg - eroded mineral ground, fe - fine earth in cracks, niches and on ledges among cliffs and rock fields, hm - humus-rich exposed soil, $\mathbf{m i}$ - cover in mires, $\mathbf{p d}$ - plants debris (including forest litter), $\mathbf{r}$ - rock surfaces and fissures, ss - silty sediments, pumice and sandstone, tb - bark on trunk base.

\begin{tabular}{|c|c|c|c|c|c|c|c|c|c|c|c|c|c|c|c|c|}
\hline \multirow[b]{2}{*}{ Species } & \multirow{2}{*}{$\begin{array}{l}\text { Altitude } \\
\text { range }\end{array}$} & \multirow{2}{*}{\multicolumn{3}{|c|}{$\begin{array}{l}\text { Altitude } \\
\text { belts } \\
\text { F D T }\end{array}$}} & \multicolumn{11}{|c|}{ Types of habitats } & \multirow{2}{*}{$\begin{array}{l}\text { Sub- } \\
\text { strate }\end{array}$} \\
\hline & & & & & 1 & 2 & 3 & 4 & 5 & 6 & 7 & 8 & 9 & & 11 & \\
\hline Abietinella abietina (Hedw.) M. I & & $\mathrm{s}$ & & & & ( & - & · & · & • & . & · & · & & & $\mathbf{r}$ \\
\hline Amblystegium serpens (Hedw.) Bruc & $600-1$ & $\mathrm{~s}$ & . & . & + & + & + & - & - & . & . & . & . & & & $\mathrm{b}, \mathrm{dw}$ \\
\hline Amphidium asiaticum $\mathrm{Sim}-\mathrm{Sim}$, Afoni & $600-1200$ & $\mathrm{r}$ & $\mathrm{r}$ & - & . & . & . & . & . & . & . & . & . & + & & $\mathbf{r}$ \\
\hline Amphidium mougeotii (Bruch \& Schim & $600-1400$ & $\mathrm{r}$ & $\mathrm{r}$ & $\mathrm{r}$ & · & · & · & · & · & . & · & · & · & + & $\cdot$ & $\mathrm{r}$ \\
\hline Anacamptodon latidens (Besch.) Brot & & $\mathrm{r}$ & · & . & + & $\cdot$ & · & · & · & · & · & $\cdot$ & · & & & b \\
\hline Andreaea rupestris $\mathrm{Hedw}$ & $600-1600$ & $\mathrm{~s}$ & $\mathrm{~s}$ & c & $\cdot$ & . & - & . & . & . & . & . & . & & & $\mathbf{r}$ \\
\hline$i i$ Mitt. & $580-6$ & s & . & . & . & . & . & . & . & . & . & . & . & + & . & $\mathbf{r}$ \\
\hline Anomobryum concinnatum (Spruce) Lindb. & $500-($ & s & $\cdot$ & . & + & · & · & · & · & · & $\cdot$ & . & . & & $\cdot$ & eg \\
\hline Anomodon minor $(\mathrm{H}$ & & r & $\cdot$ & . & $\cdot$ & $\cdot$ & · & · & . & · & · & $\cdot$ & . & + & & $\mathbf{r}$ \\
\hline *Anomodon solovjovii & 620 & $\mathrm{u}$ & . & . & & . & - & . & . & . & . & & . & + & · & $\mathbf{r}$ \\
\hline Anomodon thraustus & $540-600$ & s & . & . & + & . & . & . & . & . & . & . & . & . & . & $\mathrm{tb}, \mathrm{b}, \mathrm{r}$ \\
\hline $\begin{array}{l}\text { Anomodontopsis rugelii (Müll. Hal.) Ignatov \& Fedosov } \\
\text { (Anomodon rugelii (Müll. Hal.) Keissl) }\end{array}$ & 610 & $\mathrm{r}$ & . & & + & & · & · & · & . & . & & . & + & & $\mathbf{r}$ \\
\hline $\begin{array}{l}\text { Aquilonium plicatulum (Lindb.) Hedenäs, Schlesak \& } \\
\text { D. Quandt (Stereodon plicatulus Lindb.; Hypnum plicatulum } \\
\text { (Limdb.) A. Jaeger) }\end{array}$ & 640 & $\mathrm{r}$ & $\cdot$ & - & $\cdot$ & $\cdot$ & · & · & $\cdot$ & $\cdot$ & · & $\cdot$ & • & + & · & $\mathbf{r}$ \\
\hline $\begin{array}{l}\text { 1, Schimp. \& W. Gümbel) Loeske } \\
\text {,, Schimp. \& W. Gümbel) Broth.) }\end{array}$ & $580-1670$ & $\mathrm{r}$ & $\cdot$ & - & $\cdot$ & $\cdot$ & · & $\cdot$ & $\cdot$ & $\cdot$ & $\cdot$ & $\cdot$ & · & + & $\cdot$ & fe \\
\hline Arctoa fulvella ( & $1400-1470$ & · & · & r & · & · & · & · & $\cdot$ & · & . & + & - & · & · & fe \\
\hline $\begin{array}{r}{ }^{*} \text { Arctoa } g \\
(\text { Kiaeria }\end{array}$ & & . & . & $\mathrm{u}$ & · & . & · & . & · & · & . & + & - & $\cdot$ & . & fe \\
\hline $\begin{array}{l}\text { Arbenopter } \\
\text { beterostichum }\end{array}$ & 560 & $\mathrm{u}$ & - & - & + & $\cdot$ & · & $\cdot$ & $\cdot$ & - & $\cdot$ & · & · & $\cdot$ & $\cdot$ & dw \\
\hline & & $\mathrm{r}$ & $\cdot$ & - & + & + & $\cdot$ & $\cdot$ & - & · & . & $\cdot$ & $\cdot$ & · & · & eg \\
\hline & & c & c & - & . & + & + & + & . & . & · & - & + & · & · & mi, pd \\
\hline Aulac & & $\mathrm{r}$ & s & c & $\cdot$ & $\cdot$ & $\cdot$ & $\cdot$ & + & + & $\cdot$ & $\cdot$ & + & + & + & $\mathrm{pd}, \mathrm{fe}$ \\
\hline Bart & 150 & $\cdot$ & $\cdot$ & r & $\cdot$ & $\cdot$ & $\cdot$ & $\cdot$ & $\cdot$ & $\cdot$ & $\cdot$ & $\cdot$ & $\cdot$ & + & $\cdot$ & $r$ \\
\hline Bart & & c & s & s & $\cdot$ & $\cdot$ & $\cdot$ & $\cdot$ & $\cdot$ & $\cdot$ & $\cdot$ & $\cdot$ & $\cdot$ & + & $\cdot$ & fe \\
\hline Bar & 00 & $\mathrm{~s}$ & $\mathrm{~s}$ & s & · & + & - & - & - & - & . & - & $\cdot$ & + & - & fe, hm \\
\hline Blina & & $\mathrm{r}$ & $\mathrm{r}$ & $\mathrm{r}$ & . & . & - & . & . & . & . & - & + & + & - & $\mathbf{r}$ \\
\hline $\begin{array}{l}\text { beciastrum trachypodium (Brid.) lgnat } \\
\text { en }\end{array}$ & 1480 & $\cdot$ & $\cdot$ & $\mathrm{r}$ & $\cdot$ & $\cdot$ & · & $\cdot$ & $\cdot$ & $\cdot$ & $\cdot$ & $\cdot$ & $\cdot$ & + & $\cdot$ & fe \\
\hline ium haicalense Ionatov & 600 & $\mathrm{u}$ & $\cdot$ & - & + & $\cdot$ & · & $\cdot$ & $\cdot$ & $\cdot$ & . & - & $\cdot$ & . & - & pd \\
\hline Brach & $600-750$ & $\mathrm{r}$ & $\cdot$ & · & + & · & · & · & · & · & $\cdot$ & · & · & · & $\cdot$ & tb \\
\hline $\begin{array}{l}\text { Brachythecium campe } \\
\text { W. Gümbel }\end{array}$ & 540 & $\mathrm{u}$ & . & - & + & . & - & . & . & $\cdot$ & $\cdot$ & - & . & . & · & tb \\
\hline im cirrosum (Schwägr.) Schimp. & & $\cdot$ & $\cdot$ & u & $\cdot$ & $\cdot$ & · & · & · & · & $\cdot$ & $\cdot$ & • & + & · & fe \\
\hline${ }^{*} \mathrm{Bra}$ & & $\mathrm{r}$ & . & - & + & - & - & - & - & - & $\cdot$ & - & $\cdot$ & + & - & fe, pd \\
\hline $\begin{array}{l}\text { Brachythecium erythrorrbizon Bruch, Schimp. \& } \\
\text { W. Gümbel }\end{array}$ & $1200-1580$ & . & $\mathrm{r}$ & $\mathrm{r}$ & . & . & · & . & · & $\cdot$ & + & · & + & + & . & hm, pd \\
\hline Brac & & $\mathrm{r}$ & $\cdot$ & - & . & - & · & · & - & - & & & + & · & & $\mathrm{n}, \mathrm{pd}$ \\
\hline Brachythecium rot & 620 & $\mathrm{r}$ & . & . & + & . & · & . & . & . & . & . & . & . & . & $d w$ \\
\hline $\begin{array}{l}\text { Brideliella wablenbergii (Brid.) Fedosov, M. Stech \& Ignatov } \\
\text { (Oncophorus wablenbergii Brid.) }\end{array}$ & $600-1400$ & $\mathrm{r}$ & $\cdot$ & $\mathrm{r}$ & . & . & - & $\cdot$ & . & $\cdot$ & . & · & + & . & . & fe \\
\hline Brothera lean & 600 & $\mathrm{r}$ & $\cdot$ & - & + & + & + & · & $\cdot$ & - & · & - & $\cdot$ & $\cdot$ & - & dw \\
\hline vthrothollum of ferr & 64 & $\mathrm{r}$ & $\cdot$ & - & · & . & r & - & - & - & . & - & - & + & - & $\mathrm{fe}$ \\
\hline & & $\mathrm{r}$ & . & - & + & · & · & · & - & - & $\cdot$ & - & . & $\cdot$ & - & tb \\
\hline Bryo & $540-700$ & c & . & . & + & + & - & . & - & - & - & & • & + & & $\mathrm{fe}, \mathrm{r}$ \\
\hline Bryu & $540-700$ & s & . & - & $\cdot$ & $\cdot$ & - & - & - & - & $\cdot$ & - & + & + & - & eg, fe \\
\hline chreb.) Turner & & $\mathrm{r}$ & - & & - & - & · & - & - & - & . & - & + & · & - & \\
\hline & 185 & $\mathrm{u}$ & . & - & . & . & . & - & . & . & + & . & & . & . & eg, hm \\
\hline & $570-600$ & $\mathrm{r}$ & - & . & + & . & - & - & - & . & · & & & . & & $\mathrm{hm}$ \\
\hline *Bryum neodamense Itzigs. & & $\mathrm{u}$ & $\mathrm{u}$ & • & $\cdot$ & $\cdot$ & · & $\cdot$ & $\cdot$ & - & $\cdot$ & - & + & $\cdot$ & $\cdot$ & eg \\
\hline $\begin{array}{l}\text { Bryum pseudotriquetrum (Hedw.) G. Gaertn. B. Mey. \& } \\
\text { Scherb. }\end{array}$ & $600-1400$ & $\mathrm{~s}$ & $\mathrm{~s}$ & & . & . & $\cdot$ & + & . & . & . & & & . & & $\mathrm{hm}$ \\
\hline *Bryum weigelii Spreng. & & $\mathrm{r}$ & . & - & · & · & · & · & . & · & $\cdot$ & · & + & $\cdot$ & · & $\mathrm{mi}$ \\
\hline $\begin{array}{l}\text { *Buckia vaucheri (Lesq.) D. Ríos, M.T. Gallego \& J. Guerra } \\
\text { (Stereodon vaucheri (Lesq.) Lindb. ex Broth.; Hypmum vaucheri Lesq.) }\end{array}$ & & & $\mathrm{r}$ & . & $\cdot$ & . & . & $\cdot$ & $\cdot$ & $\cdot$ & · & · & & + & . & $\mathrm{r}, \mathrm{fe}$ \\
\hline $\begin{array}{l}\text { Bucklandiella laeta (Besch. \& Cardot) Bedn.-Ochyra \& } \\
\text { Ochyra }\end{array}$ & $600-1770$ & $\mathrm{r}$ & $\mathrm{s}$ & $\mathrm{c}$ & - & - & · & $\cdot$ & $\cdot$ & $\cdot$ & $\cdot$ & - & & + & + & $\mathbf{r}$ \\
\hline Bucklandiella microcarpa (Hedw.) Bedn.-Ochyra \& Ochyra & $1720-1770$ & & 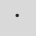 & $\mathrm{s}$ & $\cdot$ & $\cdot$ & · & $\cdot$ & · & $\cdot$ & · & $\cdot$ & · & + & + & $\mathbf{r}$ \\
\hline
\end{tabular}


Table 1. Continued.

\begin{tabular}{|c|c|c|c|c|c|c|c|c|c|c|c|c|c|c|c|c|}
\hline \multirow{2}{*}{ Species } & \multirow{2}{*}{$\begin{array}{l}\text { Altitude } \\
\text { range }\end{array}$} & \multirow{2}{*}{\multicolumn{3}{|c|}{$\begin{array}{l}\begin{array}{c}\text { Altitude } \\
\text { belts }\end{array} \\
\text { F D T }\end{array}$}} & \multicolumn{11}{|c|}{ Types of habitats } & \multirow{2}{*}{$\begin{array}{l}\text { Sub- } \\
\text { strate }\end{array}$} \\
\hline & & & & & 1 & 2 & 3 & 4 & 5 & 6 & 7 & & 9 & & 11 & \\
\hline${ }^{*}$ Bucklandiella nitidula $(\mathrm{Ca}$ & 20 & & & $\mathrm{r}$ & & $\cdot$ & & & & & & & . & + & & $\mathbf{r}$ \\
\hline Bucklandiella sudetica (Funck) B & 1740 & . & & $\mathrm{r}$ & . & . & - & . & & . & . & & . & & + & $\mathrm{r}$ \\
\hline Callicladium haldaneanum (Grev.) H.A. Crum & 580 & s & & & + & . & . & . & & . & & . & . & . & & $\mathrm{tb}, \mathrm{dw}$ \\
\hline Calliergon cordifolium (Hedw.) Kindb. & 590 & $\mathrm{r}$ & & . & $\cdot$ & . & . & . & & . & & & . & + & & \\
\hline Calliergonella lindbergii (Mitt.) Hedenäs & $560-1500$ & c & $\mathrm{r}$ & $\mathrm{r}$ & + & . & · & + & & - & & . & + & + & . & $\begin{array}{l}\text { dw, hm, } \\
\text { pd, } r\end{array}$ \\
\hline *Campyliadelphus chrysophyllus (Brid.) Kanda & 580 & $\mathrm{r}$ & · & . & & . & - & - & & . & - & - & + & . & . & ss \\
\hline * Campylium protens & & $\mathrm{r}$ & . & . & & . & . & . & . & . & . & $\cdot$ & + & · & · & mi \\
\hline Campylium stellatum & & $\mathrm{u}$ & . & . & + & . & . & . & & . & . & . & . & . & . & $\mathrm{hm}$ \\
\hline Campylophyllopsis & 560 & $\mathrm{~s}$ & . & . & + & . & . & . & & . & & . & . & + & & $d w, f e$ \\
\hline *Campylopus cf. gracilis (Mitt.) A. Jaeg & 1500 & $\cdot$ & $\mathrm{u}$ & · & & · & . & . & . & . & $\cdot$ & . & . & . & + & $\mathrm{hm}$ \\
\hline Campylopus schimperi Milc & 1770 & . & & $\mathrm{u}$ & 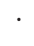 & . & · & - & . & · & $\cdot$ & · & · & + & ( & $\mathrm{fe}$ \\
\hline Ceratodon purpureus (Hedw.) Brid. & $580-1570$ & c & c & c & + & + & + & + & + & + & + & - & + & + & + & $\begin{array}{l}\text { eg, hm, } \\
\text { pd, fe }\end{array}$ \\
\hline *Cirriphyllum piliferum (Hedw.) Grout & 610 & $\mathrm{u}$ & . & . & + & - & . & . & - & . & - & . & . & . & . & hm \\
\hline Claop & & $\mathrm{r}$ & . & . & & . & . & . & . & $\cdot$ & . & . & . & + & - & $\mathrm{hm}, \mathrm{r}$ \\
\hline Climacium dendroides & 700 & $\mathrm{~s}$ & . & . & + & . & . & . & . & . & - & . & + & & . & $\mathrm{hm}, \mathrm{pd}$ \\
\hline Cnestrun & 1570 & $\mathrm{r}$ & $\mathrm{r}$ & . & . & . & + & . & . & . & . & . & . & + & . & eg \\
\hline Cosci & 650 & $\mathrm{r}$ & & . & & . & . & . & . & . & . & . & . & + & . & $\mathrm{r}$ \\
\hline${ }^{*} \operatorname{Cos}$ & 160 & . & $\mathrm{r}$ & . & · & . & . & . & . & . & . & . & . & + & . & $\mathbf{r}$ \\
\hline${ }^{*}$ Cratonen & 600 & $\mathrm{u}$ & & . & + & - & . & . & . & . & - & . & . & . & . & ss \\
\hline * Cynodontium aspe & 520 & $\mathrm{~s}$ & $\mathrm{~s}$ & $\mathrm{~s}$ & + & + & . & . & . & . & . & . & + & + & + & fe, dw \\
\hline Cynodontium s & 500 & $\mathrm{r}$ & $\mathrm{r}$ & · & & + & . & . & . & . & & . & . & . & . & fe \\
\hline Cynodont & & . & . & $\mathrm{u}$ & - & . & . & . & . & . & . & . & . & + & 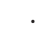 & $\mathrm{fe}$ \\
\hline Cyrtom & & . & $\mathrm{u}$ & & . & . & . & . & . & $\cdot$ & . & . & . & + & - & $\mathrm{fe}$ \\
\hline Dichodontium p & & s & $\mathrm{s}$ & · & . & · & . & . & . & . & $\cdot$ & . & + & · & · & eg, $r$ \\
\hline Dicra & & $\mathrm{r}$ & . & . & + & . & . & . & . & . & . & . & + & . & . & eg \\
\hline${ }^{*} \mathrm{Dic}$ & 600 & $\mathrm{~s}$ & $\mathrm{~s}$ & $\mathrm{~s}$ & + & . & . & . & . & . & . & . & . & + & . & $\mathrm{hm}, \mathrm{fe}$ \\
\hline Dicranella sp. & & $\mathrm{u}$ & . & . & . & - & . & . & . & . & - & . & + & . & . & ss \\
\hline Dicr & & . & $\mathrm{r}$ & $\mathrm{r}$ & . & . & . & . & + & + & . & . & . & . & . & eg \\
\hline Dicr & 580 & $\mathrm{r}$ & . & . & . & . & . & . & . & . & . & . & + & & . & ss \\
\hline Dicr & 560 & $\mathrm{r}$ & $\mathrm{r}$ & . & + & + & . & . & . & . & . & . & . & . & & dw \\
\hline Dicr & & $\mathrm{s}$ & $\mathrm{s}$ & . & + & + & . & $\cdot$ & . & $\cdot$ & . & · & . & . & • & dw \\
\hline . Jensen & & . & $\mathrm{u}$ & . & . & . & . & + & . & . & . & . & . & . & - & $\mathrm{hm}$ \\
\hline Dicr & & . & $\mathrm{r}$ & . & . & . & . & + & . & . & . & . & . & . & . & $\mathrm{mi}$ \\
\hline Dicr & 770 & . & $\mathrm{r}$ & $\mathrm{r}$ & . & . & . & + & . & . & . & . & . & . & & mi, pd \\
\hline *Dicranum drum & & $\mathrm{r}$ & . & & . & . & . & + & . & . & . & . & . & . & & pd \\
\hline Dicra & & . & $\mathrm{r}$ & $\mathrm{r}$ & . & . & . & . & . & + & . & . & . & + & + & hm, pd \\
\hline Dic & & s & & . & + & + & + & . & · & . & · & . & . & & . & tb, \\
\hline Dic & & $\mathrm{u}$ & . & . & . & . & + & . & . & . & . & . & . & & . & $\mathrm{hm}$ \\
\hline Dicr & & $\mathrm{s}$ & . & . & + & - & . & . & . & . & & - & . & & . & $\mathrm{dw}$ \\
\hline Dicr & & $\mathrm{s}$ & . & . & + & . & + & . & . & . & . & . & . & . & . & $\mathrm{tb}, \mathrm{dw}$ \\
\hline Dict & & $\mathrm{r}$ & $\mathrm{r}$ & . & + & + & . & . & . & . & . & . & . & . & . & dw \\
\hline${ }^{*} \mathrm{Dic}$ & & $\mathrm{r}$ & $\mathrm{r}$ & . & + & . & . & $\cdot$ & + & . & . & . & . & . & . & hm, dw \\
\hline${ }^{*}$ Dicr & & $\mathrm{u}$ & . & . & . & - & . & + & . & . & . & . & . & . & . & $\mathrm{mi}$ \\
\hline Dicranum & & c & c & $\mathrm{c}$ & + & + & . & . & + & . & . & . & . & + & & hm, pd \\
\hline Dic & & $\mathrm{r}$ & . & . & + & + & + & . & . & . & . & . & . & & . & \\
\hline & & . & . & $\mathrm{r}$ & . & . & . & . & . & + & . & . & . & & & $\mathrm{hm}$ \\
\hline Dicr & & . & . & $\mathrm{r}$ & & . & . & . & . & + & & - & . & & & $\mathrm{pd}, \mathrm{hm}$ \\
\hline & & $\mathrm{s}$ & . & . & + & . & . & . & & . & & . & . & & & $\mathrm{dw}, \mathrm{pd}$ \\
\hline Dic & & . & $\mathrm{u}$ & . & . & . & . & . & . & . & . & . & . & & + & fe \\
\hline Dic & $600-1570$ & $\mathrm{r}$ & $\mathrm{r}$ & $\mathrm{r}$ & . & . & + & . & + & . & . & . & . & + & & $\mathrm{hm}$ \\
\hline Dicranum undulat & & $\mathrm{u}$ & & & & . & . & + & & $\cdot$ & & · & . & & & pd \\
\hline $\begin{array}{l}\text { Dilutineuron brevisetum (Lindb.) Bedn.-Ochyra, Sawicki, } \\
\text { Ochyra, Szczecińska \& Plášek }\end{array}$ & $600-1770$ & s & s & $\mathrm{s}$ & . & . & . & . & & . & & . & + & + & & $\mathrm{r}, \mathrm{fe}$ \\
\hline $\begin{array}{l}\text { Dilutineuron fasciculare (Hedw.) Bedn.-Ochyra, Sawicki, } \\
\text { Ochyra, Szczecińska \& Plášek }\end{array}$ & 1200-1770 & & $\mathrm{s}$ & $\mathrm{s}$ & & . & . & - & & - & & & + & + & & \\
\hline $\begin{array}{l}\text { Distichium capillaceum (Hedw.) Bruch, Schimp. \& } \\
\text { W. Gümbel }\end{array}$ & 600 & $\mathrm{r}$ & & & + & - & & - & & - & & & + & & & fe, ss \\
\hline allum (Hedw.) E. Britton & & $\mathrm{r}$ & & & & . & . & . & & . & . & . & . & + & & fe \\
\hline *Ditri & & $\mathrm{u}$ & . & . & & . & . & . & . & . & & . & + & & & ss \\
\hline & & $\mathrm{r}$ & · & & & · & . & . & & . & & & + & & & dw, ss \\
\hline & $570-1400$ & $\mathrm{u}$ & $\mathrm{u}$ & & + & . & . & . & & . & & . & . & & & pd \\
\hline Encalypta ciliata Hedw. & $560-660$ & $\mathrm{r}$ & 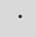 & . & & . & . & . & . & . & & . & . & + & & fd, eg \\
\hline Entodon schleicheri (Bruch, Schimp. \& W. Gümbel) Demet. & & $\mathrm{u}$ & . & . & & . & . & . & . & & & & . & & & $\mathrm{fe}^{\circ}$ \\
\hline & $560-600$ & $\mathrm{r}$ & & & + & . & . & . & & & & & . & & & $\mathrm{dw}, \mathrm{tb}, \mathrm{r}$ \\
\hline & 540 & $\mathrm{r}$ & & & + & . & - & . & & & & & . & & & \\
\hline Eurhynchiastrum pulchellum (Hedw.) Ignatov \& Huttunen & $540-700$ & c & & $\cdot$ & + & - & · & · & e & · & $\cdot$ & $\cdot$ & . & + & & $\begin{array}{l}\text { fe, } \\
\text { tb }\end{array}$ \\
\hline $\begin{array}{l}\text { Fabronia ciliaris (Brid.) Brid. } \\
\text { Fissidens adianthoides Hedw }\end{array}$ & $600-1$ & $\begin{array}{l}\mathrm{r} \\
\mathrm{u}\end{array}$ & $\mathrm{r}$ & . & + & . & . & . & $\dot{.}$ & . & - & . & . & + & & $\begin{array}{c}\mathbf{r} \\
\mathrm{hm}\end{array}$ \\
\hline
\end{tabular}


Table 1. Continued.

\begin{tabular}{|c|c|c|c|c|c|c|c|c|c|c|c|c|c|c|c|c|c|}
\hline \multirow{2}{*}{ Species } & \multirow{2}{*}{$\begin{array}{l}\text { Altitude } \\
\text { range }\end{array}$} & \multicolumn{3}{|c|}{$\begin{array}{l}\text { Altitude } \\
\text { belts }\end{array}$} & \multicolumn{12}{|c|}{ Types of habitats } & \multirow{2}{*}{$\begin{array}{l}\text { Sub- } \\
\text { strate }\end{array}$} \\
\hline & & & & $T$ & 1 & 2 & 3 & & 4 & 5 & 6 & 7 & 8 & 9 & 10 & 11 & \\
\hline Fissidens cf. bryoides Hedw. & $540-600$ & $\mathrm{r}$ & & • & · & + & • & & - & & - & & - & & + & & $\mathrm{fe}, \mathrm{r}$ \\
\hline Fissidens dubius $\mathrm{P} . \mathrm{Beauv}$. & $540-1100$ & s & $\mathrm{r}$ & & + & + & $\cdot$ & & . & & - & • & - & - & + & . & hm, eg, \\
\hline Fissidens gymnogynus Besch. & $560-645$ & c & . & & + & . & + & & + & 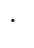 & . & · & · & $\cdot$ & + & . & $\mathrm{hm}$ \\
\hline Funaria hygrometrica Hedw. & & s & · & & + & $\cdot$ & $\cdot$ & & . & . & · & · & · & + & $\cdot$ & · & fe \\
\hline * Glyphomitrium crispifol & $620-1480$ & $\mathrm{~s}$ & s & . & + & + & + & & & & $\cdot$ & & $\cdot$ & $\cdot$ & $\cdot$ & $\cdot$ & $b$ \\
\hline Gollania ruginosa (Mitt.) Broth. & $540-1770$ & $\mathrm{~s}$ & $\mathrm{r}$ & & + & + & . & & & & $\cdot$ & & $\cdot$ & & + & & hm, pd, \\
\hline Grimmia cf. hartmanii Schimp. & 1600 & & $\mathrm{u}$ & & · & . & - & & - & & $\cdot$ & + & . & & $\cdot$ & & fe \\
\hline Grimmia donniana $\mathrm{S}$ & $1400-1770$ & & . & $\mathrm{r}$ & - & . & . & & - & - & . & & . & . & + & + & $\mathbf{r}$ \\
\hline vägr.) Bruch, Schimp. \& W. Gümbel & 1600 & & · & $\mathrm{u}$ & · & · & $\cdot$ & & · & & $\cdot$ & . & . & & $\cdot$ & + & $\mathbf{r}$ \\
\hline Grimmia incurt & 15 & & · & $\mathrm{r}$ & · & · & $\cdot$ & & . & . & $\cdot$ & . & . & . & + & + & $\mathbf{r}$ \\
\hline Grimmia lon & $540-$ & $\mathrm{s}$ & & & · & · & $\cdot$ & & . & . & · & · & · & + & + & $\cdot$ & $\mathbf{r}$ \\
\hline Grimmia p & $640-1570$ & c & c & . & . & . & . & & - & . & . & - & - & . & + & · & $\mathbf{r}$ \\
\hline Grimmia & $1200-1$ & & & $\mathrm{~s}$ & . & . & & & . & & . & . & . & & + & + & $\mathbf{r}$ \\
\hline tifolium (Hampe \& Müll. Hal.) Broth. & 690 & $\mathrm{r}$ & · & · & + & + & + & & · & & · & · & $\cdot$ & · & + & $\cdot$ & r, dw \\
\hline icrophyllum (Hedw.) Broth. & 645 & $\mathrm{u}$ & . & · & · & . & $\cdot$ & & . & & · & · & $\cdot$ & · & + & $\cdot$ & $\mathbf{r}$ \\
\hline Broth.) Broth. & $640-1720$ & $\mathrm{~s}$ & $\mathrm{~s}$ & . & - & . & . & & - & & - & - & - & . & + & . & $\mathbf{r}$ \\
\hline Haplohymenium triste (Ces.) I & $640-700$ & $\mathrm{r}$ & & · & · & · & . & & - & & · & · & · & . & + & $\cdot$ & $\mathbf{r}$ \\
\hline echinata Ignatova \& Ignatov & & s & · & · & · & · & - & & • & & · & · & $\cdot$ & · & + & + & $\mathbf{r}$ \\
\hline r. emodica Hampe ex Müll. Hal. & & $\mathrm{r}$ & . & . & . & . & • & & • & & . & . & . & . & . & + & $\mathbf{r}$ \\
\hline Herpet & 600 & $\mathrm{r}$ & . & . & - & . & - & & & & . & . & - & . & + & $\cdot$ & $\mathbf{r}$ \\
\hline Her & 620 & $\mathrm{r}$ & . & . & . & . & + & & & & . & & . & . & . & . & $d w$ \\
\hline $\begin{array}{l}\text { Heterophyllium nemorosum (W.D.J. Koch ex Brid.) Kindb. } \\
\text { (Heterophyllium affine (Hook.) M. Fleisch.) }\end{array}$ & $540-1500$ & s & $\mathrm{s}$ & · & + & + & + & & - & & $\cdot$ & ( & · & . & + & . & dw, pd \\
\hline $\begin{array}{l}\text { Homalia trichomanoides (Hedw.) Bruch, Schimp. \& } \\
\text { W. Gümbel }\end{array}$ & $560-1500$ & c & c & · & + & + & - & & • & & $\cdot$ & - & - & - & + & . & $\mathrm{b}, \mathrm{tb}, \mathrm{r}$ \\
\hline ium connexum (Cardot) Broth. & 630 & $\mathrm{u}$ & . & . & + & . & . & & • & & . & - & . & . & . & . & dw \\
\hline *Hondaella caper & 570 & $\mathrm{u}$ & . & . & + & . & · & & • & & . & . & . & . & . & . & tb \\
\hline $\begin{array}{l}\text { Hygrohypnella ochracea (Turner ex Wilson) Ignatov \& } \\
\text { Ignatova }\end{array}$ & 600 & $\mathrm{r}$ & · & · & . & $\cdot$ & $\cdot$ & & • & . & $\cdot$ & . & . & + & . & · & $\mathbf{r}$ \\
\hline $\begin{array}{l}\text { Hygrohypnella polaris (Lindb.) Ignatov \& Ignatova } \\
\text { (Hygrobypnum polare (Lindb.) Loeske) }\end{array}$ & $540-1400$ & c & c & - & - & - & . & & " & F & $\cdot$ & " & - & + & . & . & $\mathbf{r}$ \\
\hline $\begin{array}{l}\text { Hylocomiadelphus triquetrus (Hedw.) Ochyra \& Stebel } \\
\text { (Rhytidiadelphus triquetrus (Hedw.) Warnst.) }\end{array}$ & $540-700$ & c & · & . & + & - & + & & - & Fe & $\cdot$ & - & - & · & + & · & hm, pd \\
\hline Hylocomiastrum pyrenc & 1580 & & $\mathrm{r}$ & & & - & & & & & & + & & & & & $\mathrm{hm}$ \\
\hline $\begin{array}{l}\text { Hylocomium splendens (Hedw.) Bruch, Schi } \\
\text { W. Gümbel }\end{array}$ & $540-$ & c & c & c & + & + & + & & + & + & + & ( & - & . & + & + & pd \\
\hline Hymenoloma crispulum (Hedw.) Ochyra & $1400-1600$ & & $\mathrm{r}$ & $\mathrm{r}$ & & - & · & & • & & . & - & . & - & + & . & $\mathrm{r}$ \\
\hline Hypnum cupressiforme Hedw. & 540 & c & c & c & - & . & $\cdot$ & & - & - & $\cdot$ & . & - & . & + & . & $r$ \\
\hline Hypnum cupressiforme var. subjulaceum Molendo & $540-1400$ & c & s & - & + & + & + & & & + & . & • & $\cdot$ & . & + & . & $\begin{array}{l}\mathrm{pd}, \mathrm{hm} \text {, } \\
\mathrm{dw}\end{array}$ \\
\hline Hyрпи & 1570-1770 & & $\mathrm{s}$ & . & . & . & . & & • & & + & - & . & . & + & . & r \\
\hline $\begin{array}{l}\text { Isopterygiella alpicola ( } \\
\text { (Isopterygiopsis alpicola (I }\end{array}$ & & $\mathrm{u}$ & & · & - & . & · & & • & & $\cdot$ & . & . & . & + & & fe \\
\hline $\begin{array}{l}\text { Isopterygiella pulchella (Hedw.) Ign } \\
\text { (Isopterygiopsis pulchella (Hedw.) Z. I }\end{array}$ & 620 & $\mathrm{r}$ & . & . & + & . & $\cdot$ & & • & - & . & - & . & . & . & . & $\mathrm{dw}$ \\
\hline Isopt & & c & c & c & + & + & • & & • & - & . & . & - & + & + & + & $\mathrm{hm}, \mathrm{r}$ \\
\hline Iwatsukiella leucotricha (Mitt.) W.R. Buck \& H.A. Crum & & c & $\mathrm{c}$ & . & + & + & + & & • & . & . & . & . & . & + & + & $\mathrm{b}, \mathrm{tb}, \mathrm{r}$ \\
\hline $\begin{array}{l}\text { Jochenia pallescens (Hedw.) Hedenäs, Schlesak \& D. Quandt } \\
\text { (Stereodon pallescens (Hedw.) Mitt.; Hypnum pallescens (Hedw.) } \\
\text { P. Beauv.) }\end{array}$ & $540-1280$ & s & s & · & + & + & . & & . & . & $\cdot$ & . & . & $\cdot$ & . & $\cdot$ & $\mathrm{b}, \mathrm{tb}$ \\
\hline mo & $570-600$ & $\mathrm{r}$ & . & . & + & . & • & & & . & · & . & . & + & . & . & fe, $d w$ \\
\hline folium (Dicks.) Hampe & & $\mathrm{u}$ & . & · & . & . & . & & · & & . & . & . & $\cdot$ & + & $\cdot$ & fe \\
\hline Lescuraea incurvata (Hedw.) E. Lawton & 1580 & & $\mathrm{u}$ & . & . & . & . & & • & . & . & + & . & . & . & . & eg \\
\hline \& W. Gümbel) Molendo & 1740 & & . & $\mathrm{r}$ & . & · & . & & • & . & . & . & . & . & + & . & r \\
\hline & $540-1280$ & $\mathrm{c}$ & . & . & + & + & + & & • & & . & . & . & $\cdot$ & $\cdot$ & & b \\
\hline 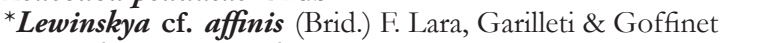 & & $\mathrm{r}$ & . & . & + & · & · & & · & · & . & . & . & . & $\cdot$ & & b \\
\hline Lewinskya cf. iwatsukii (Ignatov) F. Lara, Garilleti \& Goffinet & $550-620$ & $\mathrm{~s}$ & . & . & + & . & . & & • & . & . & . & . & . & . & . & b \\
\hline $\begin{array}{l}\text { Lewinskya elegans (Schwägr. ex Hook.\& Grev.) F. Lara, } \\
\text { Garilleti \& Goffinet }\end{array}$ & 540-920 & c & . & . & + & + & + & & • & + & $\cdot$ & & . & $\cdot$ & $\cdot$ & & b \\
\hline Lewinskya sordida (Sull. \& Lesq.) F.Lara, Garilleti \& Goffinet & $550-1500$ & $\mathrm{c}$ & c & . & + & + & + & & & + & . & · & . & . & . & & b \\
\hline & 630 & s & & & + & $\cdot$ & & & & & $\cdot$ & & . & & · & & b \\
\hline Loeskypnum badium (Hartm.) H.K.G. Paul & 1400 & $\mathrm{u}$ & $\mathrm{u}$ & . & . & · & & & 1 & & · & • & . & . & . & . & $\mathrm{mi}$ \\
\hline Mnium lycopodioides Schwägr. & $570-1500$ & s & s & . & + & . & . & & & ( & · & . & . & + & . & . & $\begin{array}{l}\text { dw, pd, } \\
\text { hm }\end{array}$ \\
\hline & & $\mathrm{u}$ & . & . & · & · & . & & & & . & • & · & · & + & & fe \\
\hline & $530-600$ & s & . & . & + & · & . & & & & . & & . & . & . & & $\mathrm{pd}, \mathrm{tb}$ \\
\hline Mnium spinulosum Bruch, Schimp. \& W. Gümbel & 600 & $\mathrm{u}$ & $\cdot$ & & . & . & • & & & & · & • & $\cdot$ & $\cdot$ & + & · & $\mathrm{fe}$ \\
\hline Mnium thomsonii Schimp. & $540-620$ & c & . & . & + & + & • & & • & • & - & - & . & · & + & . & fe, hm, \\
\hline Myurella julacea (Schwägr.) Bruch, Schimp. \& W. Gümbel & 14 & & $\mathrm{u}$ & & & · & & & & & · & & · & & 1 & & hm \\
\hline & & $\mathrm{u}$ & & & . & . & . & & & & . & . & . & . & 1 & & $\mathrm{hm}$ \\
\hline
\end{tabular}


Table 1. Continued.

\begin{tabular}{|c|c|c|c|c|c|c|c|c|c|c|c|c|c|c|c|c|}
\hline \multirow{2}{*}{ Species } & \multirow{2}{*}{$\begin{array}{l}\text { Altitude } \\
\text { range }\end{array}$} & \multicolumn{3}{|c|}{$\begin{array}{l}\text { Altitude } \\
\text { belts }\end{array}$} & \multicolumn{11}{|c|}{ Types of habitats } & \multirow{2}{*}{$\begin{array}{l}\text { Sub- } \\
\text { strate }\end{array}$} \\
\hline & & & & $T$ & 1 & 2 & 3 & 4 & 5 & 6 & 7 & 8 & 9 & 10 & 11 & \\
\hline $\begin{array}{l}\text { *Myuroclada longiramea (Müll. Hal.) Min Li,Y.F. Wan } \\
\text { Ignatov \& Huttunen }\end{array}$ & 600 & $\mathrm{u}$ & & & $\cdot$ & & • & & & & . & • & + & & & $\mathbf{r}$ \\
\hline $\begin{array}{l}\text { Myuroclada maximowiczii (G.G. Borshch.) Steere \& } \\
\text { W.B. Schofield }\end{array}$ & $540-600$ & $\mathrm{~s}$ & & & + & 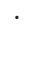 & 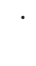 & · & & · & - & · & . & + & & fe, tb \\
\hline Neckera cf. borealis Nog. & $540-620$ & $\mathrm{~s}$ & & & + & . & - & · & & . & . & . & . & $\cdot$ & . & b \\
\hline Neckera cf. pennata Hedw. & $540-1480$ & s & · & & + & + & + & · & & · & . & · & · & $\cdot$ & $\cdot$ & b \\
\hline Neckera oligocarpa Bruch (N. pennata var. tenera Müll. Hal.) & 640 & $\mathrm{u}$ & . & & . & . & . & . & . & . & . & . & . & + & . & $\mathbf{r}$ \\
\hline Niphotrichum canescens (Hedw.) Bedn.-Ochyra \& Ochyra & 580 & $\mathrm{u}$ & · & & $\cdot$ & · & · & . & . & . & . & . & . & + & . & $\mathrm{fe}$ \\
\hline $\begin{array}{l}\text { * Niphotrichum elongatum (Ehrh. ex Frisvoll) Bedn.-Ochyra } \\
\text { \& Ochyra }\end{array}$ & $600-1780$ & s & s & c & · & · & · & $\cdot$ & $\cdot$ & + & + & + & + & $\cdot$ & + & $\mathrm{r}, \mathrm{fe}$ \\
\hline Niphotrichum panschii (Müll. Hal.) Bedn.-Ochyra \& Ochyra & $570-1570$ & c & c & & $\cdot$ & - & - & - & & - & - & $\cdot$ & . & + & + & fe \\
\hline $\begin{array}{l}\text { Nybolmiella obtusifolia (Brid.) Holmen \& Warncke } \\
\text { (Orthotrichum obtusifolium Brid.) }\end{array}$ & $54-600$ & c & & & + & . & • & · & & · & · & · & $\cdot$ & $\cdot$ & . & b \\
\hline * Okamuraea hakoniensis (Mitt.) Broth. & 600 & $\mathrm{u}$ & . & - & · & - & + & - & - & . & $\cdot$ & . & . & . & . & tb \\
\hline *Oligotrichum aligerum Mitt. & 1500 & $\cdot$ & . & $\mathrm{u}$ & · & · & · & . & · & . & $\cdot$ & + & . & $\cdot$ & $\cdot$ & fe \\
\hline Oligotrichum falcatn & 1660 & . & $\mathrm{u}$ & & . & . & . & . & . & . & . & $\cdot$ & . & . & + & fe \\
\hline icum (Hedw.) Lam. \& DC. & 600 & $\mathrm{u}$ & . & . & . & . & . & . & . & . & . & . & + & . & . & eg \\
\hline Oligotrichum parallelum (Mitt.) Kindb. & 540,1400 & $\mathrm{u}$ & $\mathrm{u}$ & · & · & · & · & . & · & · & · & $\cdot$ & + & $\cdot$ & $\cdot$ & eg \\
\hline Oncophorus virens (Hedw.) Brid. & 1200 & $\cdot$ & $\mathrm{r}$ & . & · & . & · & . & $\cdot$ & · & · & $\cdot$ & + & $\cdot$ & · & eg \\
\hline Orthotrichum & 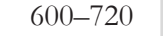 & $\mathrm{r}$ & . & . & . & + & . & . & + & . & . & . & . & . & . & b \\
\hline \&.\& Tayl.) A.J.E. Sm. & $540-1770$ & $\mathrm{c}$ & $\mathrm{c}$ & $\mathrm{r}$ & + & . & . & . & . & . & + & . & . & + & . & $\mathbf{r}$ \\
\hline *Paraleu & & & $\mathrm{u}$ & & . & . & . & . & + & . & . & . & . & + & $\cdot$ & $\mathbf{r}$ \\
\hline Paraleucobryum lon & $650-1$ & s & s & . & . & . & · & . & $\cdot$ & . & . & . & $\cdot$ & + & + & hm, pd \\
\hline Philonotis fontan & $540-650$ & $\mathrm{r}$ & . & . & . & . & . & . & . & . & . & . & + & . & . & ss \\
\hline Plagiomnium acutu & $540-600$ & c & . & . & + & . & + & . & . & . & . & . & . & . & . & hm, pd \\
\hline rnell) T.J. Kop. & 560 & $\mathrm{r}$ & . & . & + & . & . & . & . & . & . & . & . & . & . & $\mathrm{m}, \mathrm{pd}$ \\
\hline Plagiomnium c & $530-600$ & s & . & $\cdot$ & + & - & · & . & . & $\cdot$ & . & · & $\cdot$ & $\cdot$ & $\cdot$ & pd, dw \\
\hline Plagi & & $\mathrm{r}$ & . & . & . & - & - & . & . & - & . & . & + & · & . & $\mathrm{hm}$, pd \\
\hline Plagion & $600-650$ & $\mathrm{~s}$ & . & . & + & . & . & . & . & . & . & . & . & + & . & hm, pd \\
\hline Plagio & 580 & $\mathrm{~s}$ & . & . & . & . & - & . & . & . & . & . & . & + & . & $\mathrm{hm}, \mathrm{fe}$ \\
\hline $\begin{array}{l}\text { Plagiothecium denticulatum (Hedw.) Bruch, Schimp. \& } \\
\text { W. Gümbel }\end{array}$ & $1300-1500$ & $\mathrm{~s}$ & . & · & · & + & · & . & · & · & $\cdot$ & $\cdot$ & · & $\cdot$ & · & hm, pd, \\
\hline um euryphyllum (Cardot \& Ther.) Z. Iwats. & 600 & $\mathrm{r}$ & . & - & 1 & - & - & . & & - & . & $\cdot$ & . & . & . & $\mathrm{hm}$ \\
\hline *Plag & 1400 & . & $\mathrm{u}$ & . & . & . & - & . & . & . & . & . & + & . & . & $\mathrm{hm}$ \\
\hline Plagiothecium nemo & 1280 & $\mathrm{r}$ & . & · & · & + & · & $\cdot$ & · & . & · & · & $\cdot$ & $\cdot$ & . & hm, pd \\
\hline Plagiothecium svalbardense Frisvoll & & $\mathrm{s}$ & s & - & + & + & - & · & + & . & · & . & $\cdot$ & $\cdot$ & · & $\begin{array}{l}\mathrm{pd}, \mathrm{tb} \\
\mathrm{dw}\end{array}$ \\
\hline *Platydictya jungerma & & $\mathrm{u}$ & . & - & $\cdot$ & - & - & - & $\cdot$ & $\cdot$ & · & . & · & + & · & fe \\
\hline Platy & 600 & $\mathrm{~s}$ & . & · & + & . & . & . & . & . & . & . & . & . & · & tb, b \\
\hline Platy & 1200 & $\mathrm{r}$ & . & . & . & . & - & . & . & . & . & . & + & . & . & $\mathbf{r}$ \\
\hline ariifolium (Venturi) Ochyra & 1500 & $\cdot$ & $\mathrm{r}$ & & & . & $\cdot$ & $\cdot$ & · & · & $\cdot$ & $\cdot$ & + & $\cdot$ & · & $\mathrm{r}$ \\
\hline Pleurozium schreberi (Brid.) Mitt. & $540-15$ & c & c & & + & + & + & + & - & . & · & $\cdot$ & $\cdot$ & + & & $\begin{array}{l}\mathrm{pd}, \mathrm{tb} \\
\mathrm{dw}\end{array}$ \\
\hline Podperaea krylovii & & $\mathrm{u}$ & . & 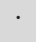 & + & . & · & · & . & - & . & $\cdot$ & . & + & . & $\mathrm{hm}$ \\
\hline Pogo & $590-$ & c & $\mathrm{c}$ & & + & · & $\cdot$ & · & · & · & + & $\cdot$ & + & + & · & $\mathrm{hm}$ \\
\hline Pogo & 590 & $\mathrm{r}$ & . & . & . & . & - & . & . & . & . & . & · & + & . & fe, eg \\
\hline Pogo & 62 & $\mathrm{u}$ & . & & + & . & . & . & . & . & . & . & . & . & . & eg \\
\hline Pogo & $530-1680$ & s & . & - & $\cdot$ & · & · & · & · & . & . & $\cdot$ & + & + & + & eg, ss \\
\hline Poblia an & & s & & & & · & $\cdot$ & · & · & · & · & $\cdot$ & + & & & eg \\
\hline *Poblia a & $128+2>$ & . & $\mathrm{r}$ & . & . & . & - & . & . & - & . & . & + & . & . & eg \\
\hline Poblia & & $\mathrm{c}$ & $\mathrm{c}$ & & + & . & . & . & & . & . & . & + & + & . & fe, hm \\
\hline Poblia & $580-1280$ & s & s & - & . & + & . & . & . & . & . & . & + & + & $\cdot$ & fe \\
\hline Müll. Hal.) A.L. Andrews & 1680 & & & $\mathrm{r}$ & & 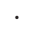 & $\cdot$ & · & · & · & + & $\cdot$ & $\cdot$ & $\cdot$ & & fe \\
\hline Poblia elon & 640 & $\mathrm{r}$ & . & & . & - & - & · & . & . & $\cdot$ & . & $\cdot$ & + & · & fe, hm \\
\hline Pobl & 580 & $\mathrm{r}$ & $\mathrm{r}$ & & & + & . & . & & . & . & . & + & + & & eg \\
\hline * Poblia ludwi & & $\cdot$ & $\mathrm{r}$ & & . & . & . & . & . & $\cdot$ & $\cdot$ & + & $\cdot$ & $\cdot$ & . & fe \\
\hline *Poblia lutesce & & $\mathrm{r}$ & & & . & • & . & . & & . & . & . & + & . & & eg, ss \\
\hline Poblia nu & $530-1$ & s & . & - & + & + & + & + & + & . & · & $\cdot$ & $\cdot$ & . & . & $\mathrm{hm}, \mathrm{dw}$ \\
\hline Poblia & 60 & $\mathrm{~s}$ & . & & + & . & . & . & & . & . & . & . & + & . & eg \\
\hline *Pobliat & $540-600$ & $\mathrm{~s}$ & . & . & . & . & . & . & & - & . & . & + & · & . & ss \\
\hline Poblia wablenbergii (F. Weber \& D. Mohr) A.L. Andrews & 570 & $\mathrm{u}$ & & & & • & . & . & & . & . & . & + & . & & ss \\
\hline Polytrichastru & $600-1600$ & s & $\mathrm{s}$ & & . & • & . & · & & . & + & . & . & + & + & fe, hm \\
\hline $\begin{array}{c}\text { * Polytrichastr } \\
\text { N.E. Bell \& }\end{array}$ & & & & $\mathrm{r}$ & & & · & · & & . & & + & & & & \\
\hline & 600 & . & $\mathrm{s}$ & & & & • & $\cdot$ & & . & $\cdot$ & . & . & . & & \\
\hline & 1400 & . & $\mathrm{r}$ & & . & + & . & . & & · & . & $\cdot$ & . & $\cdot$ & & $\mathrm{hm}$ \\
\hline niperinum Hedw. & $560-1570$ & s & s & . & . & . & · & · & · & . & . & . & . & + & + & eg \\
\hline 4 & 620 & $\mathrm{u}$ & . & & . & . & + & & & . & . & . & . & . & . & hm, pd \\
\hline & $1500-1580$ & . & $\mathrm{r}$ & & . & · & . & + & & . & . & . & . & + & + & eg, fe \\
\hline Polytrichum s & 540-610 & $\mathrm{r}$ & • & & . & - & - & + & & . & . & . & . & $\cdot$ & . & pd \\
\hline $\begin{array}{l}\text { *Pseudoamblystegium subtile (Hedw.) Vanderp. \& Hedenäs } \\
\text { (Serpoleskea subtilis (Hedw.) Loeske) }\end{array}$ & 600 & $\mathrm{u}$ & . & & . & & . & · & . & . & . & . & . & + & . & $r$ \\
\hline
\end{tabular}


Table 1. Continued.

\begin{tabular}{|c|c|c|c|c|c|c|c|c|c|c|c|c|c|c|c|c|}
\hline \multirow[b]{2}{*}{ Species } & \multirow{2}{*}{$\begin{array}{l}\text { Altitude } \\
\text { range }\end{array}$} & \multirow{2}{*}{\multicolumn{3}{|c|}{$\begin{array}{l}\begin{array}{c}\text { Altitude } \\
\text { belts }\end{array} \\
\text { F D T }\end{array}$}} & \multicolumn{11}{|c|}{ Types of habitats } & \multirow{2}{*}{$\begin{array}{l}\text { Sub- } \\
\text { strate }\end{array}$} \\
\hline & & & & & 1 & 2 & 3 & 4 & 5 & 6 & 7 & 8 & 9 & 10 & 11 & \\
\hline $\begin{array}{l}\text { *Pseudoblindia falcata (Hedw.) Fedosov, M. Stech \& Ignatov } \\
\text { (Kiaeria falcata (Hedw.) I. Hagen) }\end{array}$ & 1740 & & & $\mathrm{u}$ & - & & & & & & & + & & & & $\mathbf{r}$ \\
\hline $\begin{array}{l}\text { Pseudohygrohypnum cf. subeugyrium (Renauld \& Cardot) } \\
\text { Ignatov \& Ignatova }\end{array}$ & $580-1300$ & $\mathrm{~s}$ & $\mathrm{~s}$ & & & & & & & & & & + & & & $\mathbf{r}$ \\
\hline $\begin{array}{l}\text { Pseudohygrohypnum fauriei (Cardot) Jan Kučera \& Ignatov } \\
\text { (Stereodon fauriei (Cardot) Afonina; Hypnum fauriei Cardot) }\end{array}$ & 1500 & & $\mathrm{u}$ & . & . & . & . & & re $>2+2>$ & . & & . & . & . & + & $\mathbf{r}$ \\
\hline Ptilium crista-castrensis (Hedw.) De Not. & $520-1720$ & c & s & s & + & + & + & • & . & + & & · & . & + & + & $\underset{\mathrm{tb}}{\mathrm{pd}, \mathrm{dw}}$ \\
\hline Pylaisia condensata (Mitt.) A. Jaeger & $520-600$ & c & . & . & + & . & . & & & . & & . & . & . & . & b \\
\hline Pylaisia polyantha (Hedw.) Bruch, S & $520-600$ & c & . & . & + & . & . & • & . & . & . & . & . & . & . & b \\
\hline $\begin{array}{l}\text { Pylaisiadelpha tenuirostris (Bruch \& Schimp. ex Sull.) } \\
\text { W.R. Buck }\end{array}$ & $560-1570$ & s & $\mathrm{r}$ & . & + & . & . & . & . & $\cdot$ & . & . & . & + & $\cdot$ & dw, tb \\
\hline $\begin{array}{l}\text { * Pylaisiadelpha tristoviridis (Broth.) Afonina, H. Tsubota } \\
\text { \& Ignatova }\end{array}$ & 570 & $\mathrm{r}$ & & . & + & • & . & • & & . & & . & . & . & . & b \\
\hline Racomitrium lanuginosum (Hedw.) Brid. & $1500-1720$ & & $\mathrm{~s}$ & $\mathrm{~s}$ & . & . & . & . & . & . & . & . & . & + & + & $\mathrm{r}, \mathrm{eg}$ \\
\hline Rauiella fujisana (Paris) Reimers & 560 & $\mathrm{r}$ & . & . & + & . & . & . & . & . & . & . & . & . & . & b \\
\hline Rhabdoweisia crispata (Dicks. & $600-1570$ & s & s & . & . & . & . & . & . & . & . & . & . & + & + & fe \\
\hline Rhizomnium magnifolium (Horik.) T.J. Kop. & 540 & $\mathrm{r}$ & . & . & + & . & . & . & . & . & . & . & . & $\cdot$ & . & hm \\
\hline Rhizomnium striatulum (Mitt.) T.J. Kop. & $540-600$ & $\mathrm{r}$ & . & . & + & . & . & . & . & . & . & . & . & + & . & hm, pd \\
\hline Rbizomnium $t$ & $570-645$ & $\mathrm{r}$ & . & . & + & . & . & . & . & . & . & . & . & + & . & $\mathrm{hm}, \mathrm{pd}$ \\
\hline Rhod & 610 & $\mathrm{r}$ & . & . & . & . & . & . & . & . & . & . & . & + & . & $\mathrm{hm}$ \\
\hline Rhodobryum rose & $570-620$ & s & . & . & + & . & . & . & . & . & . & . & . & . & . & hm, pd \\
\hline Rhytidiadelphus subpinnat & $540-570$ & $\mathrm{r}$ & . & . & + & . & . & . & . & . & . & . & . & . & . & pd \\
\hline Rhytidium rugosum (Hedw.) Kindb. & $540-1720$ & $\mathrm{c}$ & $\mathrm{c}$ & . & . & . & . & . & . & + & . & . & . & + & + & $\mathrm{r}, \mathrm{fe}$ \\
\hline Saelania glaucescens & $540-700$ & s & & . & + & . & . & . & . & . & & . & + & + & . & eg \\
\hline Sanionia uncinata (Hedw.) Loeske & & c & c & c & + & + & + & . & + & + & & + & + & + & + & $\begin{array}{l}\text { mi, hm, } \\
\text { pd, r }\end{array}$ \\
\hline Schistidium agassizii Sull. \& Lesq. & $540-610$ & $\mathrm{r}$ & . & . & . & . & . & . & . & . & - & . & + & . & . & r \\
\hline${ }^{*}$ Schistidium austrosibiricum I & 600 & $\mathrm{r}$ & . & . & . & . & . & . & . & . & . & . & . & + & . & $\mathbf{r}$ \\
\hline Schistidium lancifolium (Kindb.) H.H. Blom & 610 & $\mathrm{u}$ & . & . & . & . & . & . & $\cdot$ & $\cdot$ & . & . & . & + & $\cdot$ & $\mathbf{r}$ \\
\hline *Schistidium papillosum & $580-1480$ & $\mathrm{r}$ & $\mathrm{r}$ & . & . & . & . & . & . & . & . & . & . & + & . & $\mathbf{r}$ \\
\hline *Schistidium platvt & 580 & $\mathrm{r}$ & . & . & . & . & . & . & . & . & . & . & + & . & . & $\mathrm{r}$ \\
\hline * Schistidium sibiricum & $630-1200$ & $\mathrm{r}$ & . & . & . & . & . & . & . & . & . & . & + & . & . & $\mathbf{r}$ \\
\hline * Schistidium sordidum & $600-1400$ & $\mathrm{r}$ & $\mathrm{r}$ & . & . & . & . & . & . & . & . & . & + & . & . & $\mathbf{r}$ \\
\hline Schistostega pennata & 620 & $\mathrm{r}$ & . & . & + & . & . & . & . & . & . & . & . & . & . & eg \\
\hline Sciu & 560 & $\mathrm{r}$ & . & . & + & . & . & . & . & . & . & . & . & . & . & hm, pd \\
\hline Sciuro-bypnum plumo & $580-1200$ & $\mathrm{~s}$ & $\mathrm{~s}$ & . & . & . & . & . & . & . & . & . & + & . & . & r \\
\hline Sciuro-hypnum reflexum (Starl & $600-1500$ & s & s & . & + & . & . & . & . & . & . & . & + & . & . & hm, tb \\
\hline Sciuro-bypnum & $600-1280$ & $\mathrm{~s}$ & . & . & + & + & + & - & . & . & . & . & . & . & . & hm, pd \\
\hline * Sematophyllum & 540 & $\mathrm{u}$ & . & . & + & . & . & . & . & . & . & . & + & . & . & $\mathrm{r}, \mathrm{hm}$ \\
\hline Sphagnum alaskense R.E. Andrus \& Janssens & $600-1770$ & s & s & s & . & . & . & + & . & . & . & . & . & + & + & $\mathrm{r}, \mathrm{fe}, \mathrm{mi}$ \\
\hline $\begin{array}{l}\text { Sphagnum angustifolium (C.E.O. Jensen ex Russow) } \\
\text { C.E.O. Jensen }\end{array}$ & 600 & $\mathrm{r}$ & . & . & . & . & . & + & . & . & . & . & . & + & . & $\mathrm{r}, \mathrm{mi}$ \\
\hline . & & $\mathrm{r}$ & $\mathrm{r}$ & . & . & . & . & . & . & . & . & . & + & . & + & $\mathrm{r}, \mathrm{eg}$ \\
\hline R.E.Andrus \& B.Shaw & 610 & $\mathrm{r}$ & . & . & . & $\cdot$ & . & . & $\cdot$ & . & . & . & + & . & $\cdot$ & $\mathrm{r}, \mathrm{eg}$ \\
\hline Sphagni & 150 & & $\mathrm{r}$ & . & . & . & . & . & $\cdot$ & . & . & . & $\cdot$ & . & + & $\mathrm{r}, \mathrm{eg}$ \\
\hline * Sphagn & 610 & $\mathrm{r}$ & . & . & . & . & . & . & . & . & . & . & + & . & . & r, eg \\
\hline Sphagnu & 1500 & & $\mathrm{r}$ & . & . & . & . & . & . & . & . & . & . & . & + & r, eg \\
\hline Sphagnum divin & 62 & $\mathrm{r}$ & . & . & . & . & . & + & . & . & . & . & . & . & . & $\mathrm{mi}$ \\
\hline Sphagnu & $610-1400$ & $\mathrm{r}$ & $\mathrm{r}$ & . & . & . & . & + & . & . & . & . & . & . & . & $\mathrm{mi}$ \\
\hline Sphagn & 1400 & & $\mathrm{r}$ & . & . & . & . & + & . & . & . & . & . & . & . & $\mathrm{mi}$ \\
\hline Sphagnum fuscu & 610 & $\mathrm{r}$ & . & . & . & . & . & + & & . & . & . & . & . & . & $\mathrm{mi}$ \\
\hline Sphagnum girgensobnii Russow & $600-1500$ & $\mathrm{~s}$ & $\mathrm{~s}$ & . & . & . & + & + & . & . & . & . & . & + & + & $\mathrm{mi}, \mathrm{r}, \mathrm{eg}$ \\
\hline *Sphagnum mivabean & 620 & $\mathrm{r}$ & & . & . & . & . & + & & . & . & . & . & . & . & mi \\
\hline ium (Lindb. ex Braithw.) Warns & 600 & $\mathrm{r}$ & . & . & . & . & . & . & . & . & . & . & + & . & . & eg \\
\hline * Sphagnum rubellum Wilson & 1400 & & $\mathrm{r}$ & & . & . & . & + & . & . & . & . & . & . & . & $\mathrm{mi}$ \\
\hline * Sphagnum rubiginos & $600-1700$ & $\mathrm{r}$ & . & . & . & . & + & + & . & . & . & . & . & . & . & $\mathrm{mi}$ \\
\hline Sphagnum russow & 1500 & & $\mathrm{r}$ & . & . & . & . & $r^{-}$ & . & . & . & . & . & . & + & r \\
\hline Sphagn & $540-1280$ & $\mathrm{~s}$ & s & & . & + & . & . & & . & . & . & + & . & & eg \\
\hline sow \& Warnst. & 1570 & & $\mathrm{u}$ & & . & . & . & & & . & & . & & + & & r \\
\hline Sphagnum teres (Schimp.) Ângstr. & $600-620$ & $\mathrm{~s}$ & & . & . & . & . & + & & . & & . & + & . & . & mi \\
\hline *Sphagnum tescorum Flatberg & 630 & $\mathrm{r}$ & . & . & . & . & . & + & . & . & & . & . & . & & mi \\
\hline & 600 & $\mathrm{~s}$ & . & . & . & . & . & + & . & . & . & . & + & + & & $\mathrm{mi}, \mathrm{pd}$ \\
\hline $\begin{array}{l}\text { * Streblotrichum convolutum (Hedw.) P. Beauv. (Barbula } \\
\text { convoluta Hedw.) }\end{array}$ & 580 & $\mathrm{u}$ & . & . & . & . & . & 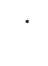 & . & . & & . & + & & . & ss \\
\hline *Struckia enervis (Brot & & $\mathrm{r}$ & $\mathrm{r}$ & & & & & & & . & & . & & + & & $r, f e, d w$ \\
\hline $\begin{array}{l}\text { Symblepharis cf. elongata (I. Hagen) Fedosov, M. Stech \& } \\
\text { Ignatov (Oncophorus elongatus (I. Hagen) Hedenäs) }\end{array}$ & 560-1280 & c & & & + & + & + & & & . & & . & & . & . & \\
\hline Syntrichia pagor & $640-690$ & $\mathrm{r}$ & . & & . & . & . & . & . & . & . & . & . & + & . & $\mathrm{r}, \mathrm{fe}$ \\
\hline & 620 & $\mathrm{u}$ & . & . & . & . & . & . & . & . & & . & . & + & . & $\mathrm{hm}$ \\
\hline * Taxiphyllum aomoriense (Besch.) & 540 & $\mathrm{r}$ & . & & + & . & . & . & & . & & . & . & . & & tb \\
\hline
\end{tabular}


Table 1. Continued.

\begin{tabular}{|c|c|c|c|c|c|c|c|c|c|c|c|c|c|c|c|}
\hline \multirow{2}{*}{ Species } & \multirow{2}{*}{$\begin{array}{l}\text { Altitude } \\
\text { range }\end{array}$} & \multirow{2}{*}{\multicolumn{2}{|c|}{$\begin{array}{l}\text { Altitude } \\
\text { belts } \\
\text { F D T }\end{array}$}} & \multicolumn{11}{|c|}{ Types of habitats } & \multirow{2}{*}{$\begin{array}{l}\text { Sub- } \\
\text { strate }\end{array}$} \\
\hline & & & & 7 & 1 & 2 & 3 & 4 & 5 & 6 & 7 & 8 & 9 & 1011 & \\
\hline $\begin{array}{l}\text { Tetraphis geniculata Girg. ex Milde } \\
\text { Tetraphis pellucida Hedw. } \\
\text { Tetraplodon mnioides (Hedw.) Bruch, Schimp. \& W. Gümbel }\end{array}$ & $\begin{array}{l}600 \\
540-1480 \\
600\end{array}$ & $\begin{array}{l}\mathrm{u} \\
\mathrm{s} \\
\mathrm{u}\end{array}$ & $\dot{.}$ & & + & + & $\dot{\cdot}+$ & $\cdot$ & $\cdot$ & $\cdot$ & . & . & $\cdot$ & $\dot{r} \cdot$ & $\begin{array}{l}\text { dw } \\
\text { dw } \\
\text { hm }\end{array}$ \\
\hline Thuidium assimile (Mitt.) A. Jaeger & $540-620$ & c & & & + & & . & . & & - & & & & + & pd, $\mathrm{dw}_{\mathrm{tb}}$ \\
\hline Thuidium delicatulum (Hedw.) Bruch, Schimp. \& W. Gümbel & $570-600$ & $\mathrm{r}$ & . & & + & & . & + & & - & & & & · & pd, dw, \\
\hline Tomentypnum cf. nitens (Hedw.) Loeske & $600-1400$ & $\mathrm{u}$ & $\mathrm{u}$ & & · & · & · & + & $\cdot$ & . & $\cdot$ & & · & · & $\mathrm{mi}$ \\
\hline Trachycystis ussuriensis (Maack \& Regel) T.J. Kop. & $540-650$ & c & . & & + & · & · & $\cdot$ & · & . & . & $\cdot$ & . & · & $\underset{\text { tb }}{h m}$, \\
\hline $\begin{array}{l}\text { *Trematodon ambiguus (Hedw.) Hornsch. } \\
\text { Trichodon cylindricus (Hedw.) Schimp. (Ditrichum cylindricum } \\
\text { (Hedw.) Grout) }\end{array}$ & $\begin{array}{c}540 \\
540-580\end{array}$ & $\begin{array}{l}\mathrm{u} \\
\mathrm{r}\end{array}$ & . & (1) & $\cdot$ & · & . & $\cdot$ & $\cdot$ & . & - & $\cdot$ & $\begin{array}{l}+ \\
+\end{array}$ & . & $\begin{array}{l}\text { ss } \\
\text { ss }\end{array}$ \\
\hline *Ulota cf. crispa Bruch & $570-620$ & $\mathrm{u}$ & . & & + & • & . & . & & - & - & . & . & . & b \\
\hline Ulota cf. intermedia Schimp. & 1300 & . & $\mathrm{r}$ & & & · & . & . & + & . & - & • & . & $\cdot$ & b \\
\hline Ulota curvifolia (Wahlenb.) Sw. & 1500 & . & $\mathrm{r}$ & & & . & . & . & & . & . & $\cdot$ & . & + & $\mathbf{r}$ \\
\hline Ulota rehmannii Jur. & $570-1300$ & $\mathrm{c}$ & . & & + & + & + & . & & . & . & $\cdot$ & . & . & b \\
\hline Ulota reptans Mitt. & 600 & $\mathrm{u}$ & . & & + & . & . & . & . & . & . & • & . & . & b \\
\hline $\begin{array}{l}\text { Warnstorfia exannulata (Bruch, Schimp. \& W. Gümbel) } \\
\text { Loeske }\end{array}$ & 1300 & $\mathrm{r}$ & . & & & · & . & + & . & . & · & $\cdot$ & $\cdot$ & . & mi \\
\hline Warnstorfia sarmentosa (Wahlenb.) Hedenäs & $600-1400$ & $\mathrm{r}$ & $\mathrm{r}$ & & & & $\cdot$ & + & & & . & & & $\cdot$ & $\mathbf{r}$ \\
\hline *Weissia brachycarpa (Nees \& Hornsch.) Jur. & $540-620$ & $\mathrm{~s}$ & & & + & & - & & & . & & & & & $\underset{\mathrm{tb}}{\mathrm{eg}} \mathrm{hm}$ \\
\hline Weissia cf. edentula Mitt. & $580-600$ & $\mathrm{u}$ & - & & + & $\cdot$ & . & - & & $\cdot$ & $\cdot$ & $\cdot$ & & . & hm \\
\hline${ }^{*}$ Weissia controversa Hedw. & 570 & $\mathrm{r}$ & . & - & + & . & · & · & & . & · & . & + & · & eg, hm \\
\hline Zygodon sibiricus Ignatov, Ignatova, Z. Iwats. \& B.C. Tan & $560-1480$ & s & & & + & + & . & 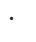 & & . & . & & & & b \\
\hline
\end{tabular}

B. recurvirostrum, Entodon sullivantii, Homalia trichomanoides, Lewinskya spp., Mnium spp., Platygyrium repens, Pylaisia condensata, Neckera cf. pennata, Zygodon sibiricus, etc. (see Table 1 in the results section). Leucodon pendulus, Lewinskya spp., Pylaisia polyantha, Jochenia pallescens, Pylaisiadelpha tenuirostris, Ulota rehmannii can be listed among the most characteristic epiphytes of coniferous trees and birch. On rotten logs suite of species from the families Brachytheciaceae, Mniaceae, Plagiotheciaceae, and Pylaisiaceae occur. In terms of ecology-floristic classification of plant cover, we preliminarily refer these communities to the association Swido albaePiceetum obovatae Krestov et Nakamura 2002, union Abieti nephrolepidis-Piceion jezoensis Song 1991, order Abieti-Piceetalia Miyawaki et al. 1968, class Vaccinio-Piceetea Br.-Bl. in Br.-B1. et al. 1939 (Krestov \& Nakamura 2002).

The described dark-coniferous forests probably represent the final stage of succession in valley-bottom, while at earlier stages of this succession, alluvial deposits in the riparian zone are occupied by various Salicaceae trees, including Salix arbutifolia, Populus suaveolens, Salix cardiophylla, and $S$. rorida. A herb layer in such riparian communities is composed of nitrophilous and hygrophilous species like Parasenecio hastatus, Impatiens noli-tangere, Mitella nuda, Thalictrum sparsiflorum, Trigonotis radicans, which alternate with spots of bare alluvium. The syntaxonomy of these riparian forests is unresolved; apparently, they belong to the class Salicetea sachalinensis Ohba 1974 (Miyawaki 1988, Kolbek et al. 2003). A moss layer is very sparse and poor in species, it is largely composed of Niphotrichum panschii, Polytrichum spp., Ceratodon purpureus, Bryoerythrophyllum recurvirostrum, Bryum argenteum, Calliergonella lindbergii, Climacium dendroides, Dichodontium pellucidum, Dicranella spp., Poblia spp., Weissia spp., Trichodon cylindricus, Philonotis fontana, Pogonatum urnigerum, and Trematodon ambiguus and other pioneer mosses as well as Plagiomnium spp., Atrichum flavisetum etc. in more shaded places. Since these species largely settle on fresh alluvium, we do not separate this type of habitat from "stream and river banks and water" (Table 1, 9), where however a suite of saxicolous mosses from the genera Aquilonium, Bucklndiella, Codriophorus, Hygrohypnella, Pseudobygrohypnum, Pylaisiadelpha, Schistidium and Sciuro-hypnum (plumosum) is better represented.

\section{Mountain dark conifer forests}

Mountain dark conifer forests on the slopes vary in composition depending on altitude. At the lowest elevation, the proportion of spruce (Piceajezoensis) and fir (Abies nephrolepis) is almost equal and Betula lanata, Larix cajanderi, Sorbus sibirica occurs as a canopy admixture. The mean canopy closure is $80 \%$; a tree layer typically consists of two sublayers, with mean heights of 20 and $10 \mathrm{~m}$. A shrub layer is up to $2 \mathrm{~m}$, usually sparse, composed of Acer ukurunduense and Lonicera maximowiczii, often absent. An herb layer consists of shade-tolerant species such as Angelica maximoniczii, Carex pallida, Diplarium sibiricum, Leptorumohra amurensis, Linnaea borealis, Listera cordata, Maianthemum bifolium, Oxalis acetosella, Phegopteris connectilis, Smilacina davurica, Vaccinium vitis-idaea, with a total cover of $30 \%$.

At higher elevations Picea jezoensis form monodominant stands or stands with admixture of Betula lanata; the canopy closure varies from 30 to $80 \%$, tree height varies from 10 to $20 \mathrm{~m}$. The shrub layer is well-developed with a mean cover of $40 \%$, composed of Alnus alnobetula subsp. fruticosa, Ledum palustre, Pinus pumila, Rhododendron aureum, Spiraea betulifolia. A herb layer with a mean cover of $20 \%$ consists of Calamagrostis purpurea, Cornus canadensis, Linnaea borealis, Maianthemum bifolium, Pedicularis kuznetzovii, Tilingia ajanensis, Vaccinium vitis-idaea. 
In the system of ecology-floristic classification, the communities can be classified into two associations of the class Vaccinio-Piceetea Br.-Bl. in Br.-Bl. et al. 1939, order AbietiPiceetalia Miyawaki et al. 1968, union Pino pumilae-Piceion jezoensis Krestov et Nakamura 2002 (Krestov \& Nakamura 2002): associations Vaccinio vitis-idaeae-Piceetum jezoensis Krestov et Nakamura 2002 and Rhododendro aurei-Piceetum jezoensis Krestov et Nakamura 2002 respectively. Composition of bryophytes in both associations is very similar; a total cover varies from 30 to $80-90 \%$; the most abundant are Hylocomium splendens, Pleurozium schreberi, Hylocomiadelphus triquetrus, Dicranum spp. etc. (Table 1). On rotten logs and stumps Heterophyllum nemorosum, Iwatsukiella leucotricha, Tetraphis pellucida, Dicranum cf. montanum and other species of this genus, Jochenia pallescens, Sanionia uncinata occur.

It should be noted that dark coniferous forests formed by spruce and fir were previously more common. However the frequent fires at the beginning and middle of the 20th century resulted in the deforestation of slopes. Moreover, due to multiple fires, the spruce and fir forests in many cases were replaced by larch and birch ones, as well as thickets of Pinus pumila and talus (Manko \& Rosenberg 1967). Furthermore, Manko (1961) considers that forest fires were the reason for treeline altitude decrease in the Badzhal Range. Larch is now occurring everywhere on mountain slopes. The increasing role of larch in forest cover formation as a result of fires has also been repeatedly pointed out based on data from the neighboring regions (Sochava 1934, Orlov 1955, Gozhev 1934), as well as in Sikhote-Alin (Rosenberg 1959) and the Amur Province (Dokhturovsky 1911, Sochava 1956).

\section{Larch forests of drained habitats}

Today larch forests (Larix cajanderi) extend the significant areas in the Badzhal Range. Indigenous and secondary communities are not always easy to distinguish. Larch is the main dominant of the tree layer, but Betula platyphylla also occurs. Abies nephrolepis and Picea jezoensis occasionally appear in sub-canopy or understory layers. The mean tree height is $15 \mathrm{~m}$, and canopy cover is $60 \%$. A shrub layer is characterized by the presence of Ledum palustre (incl. Ledum bypoleucum), Pinus pumila, Rhododendron dauricum. The mean height of shrubs is $1.3 \mathrm{~m}$, and the mean cover is $60 \%$. The cover of the herb layer usually varies from 10 to $30 \%$. Common species are Carex globularis and Vaccinium vitis-idaea. The moss layer cover is about $50 \%$; it is dominated by Hylocomium splendens and Pleurozium schreberi, typically with admixture of Ptilium crista-castrensis, Dicranum spp., Polytrichum spp., and hygrophilous mosses as Aulacomnium palustre and Sphagnum spp. We considered these communities as representing the association Ledo palustris-Laricetum cajanderi Ermakov et al. 2002, union Ledo palustris-Laricion cajanderi Ermakov et al. 2004, order Ledo palustris-Laricetalia cajanderi Ermakov in Ermakov et Alsynbayev 2004, class Vaccinio-Piceetea Br.-Bl. in Br.-Bl. et al. 1939 (Krestov et al. 2009).

\section{Larch woodlands of wetlands}

Undoubtedly, indigenous communities are Larixforested wetlands which occasionally occur in weakly drained sites in valley bottoms (Manko 1961). The mean cover of trees is $30 \%$. A shrub layer from Betula ovalifolia, Chamaedaphne calyculata, Ledum palustre, Vaccinium uliginosum, Andromeda polifolia covers about $60 \%$. In sparse herb layer (cover about $5 \%$ ) Carex globularis, Drosera rotundifolia and Vaccinium microcarpum are the most abundant. The moss layer is dense, formed mainly by Sphagnum spp. with an admixture of Dicranum spp., Aulacomnium palustre, Straminergon stramineum, Loeskypnum badium, Tomentypnum nitens; a mean cover is $90 \%$. We consider the communities in the association Chamaedaphno calyculatae-Laricetum cajanderi Krestov et al. 2009, union Ledo palustris-Laricion cajanderi Ermakov et al. 2004, order Ledo palustris-Laricetalia cajanderi Ermakov in Ermakov et Alsynbayev 2004, class Vaccinio-Piceetea Br.-B1. in Br.-Bl. et al. 1939 (Krestov et al. 2009).

\section{Other vegetation types}

The dwarf-thickets belt on the investigated territory starts from about $1400 \mathrm{~m}$ a.s.l. Large areas in the upper parts of slopes are occupied by dwarf pine (Pinus pumila) and dwarf alder (Alnus alnobetula subsp. fruticosa) thickets; both species can grow together and form mixed shrub layer, typically with sparse trees of Betula lanata. The mean canopy height is $1.52 \mathrm{~m}$, and its mean total cover exceeds $70 \%$. Under the main canopy, a second shrub layer composed of Betula ovalifolia, Juniperus sibirica, Ledum palustre, Rhododendron aureum is developed with a mean cover of $10 \%$ and a mean height of $0.7 \mathrm{~m}$. A mean total cover of the herb layer is $10 \%$, Carex rigidioides, Pedicularis labradorica, Ledum decumbens, Vaccinium vitis-idaea occur here. Moss layer with the mean cover of $20 \%$ is composed of Pleurozium schreberi, Hylocomium splendens, Sciuro-hypnum reflexum, Sanionia uncinata, Ptilium crista-castrensis, Dicranum spp., etc. The communities belong to the associations Vacinio-Pinetum pumilae Maeda et Shimazaki 1951 and Ledo-Pinetum pumilae Kobayashi 1967, union Vaccinio-Pinion pumilae Suz.-Tok. 1964, order Abieti-Piceetalia Miyawaki et al. 1968, class Vaccinio-Piceetea Br.-Bl. in Br.-Bl. et al. 1939 (Miyawaki 1988, Kolbek et al. 2003). Crooked "dwarf forests" from Betula lanata also occur near the timberline. The composition of their herb layer is very similar to ones of mountain spruce-fir forests, but the set of mosses is meager. The crooked birch forests are considered in the class BetuloRanunculetea Ohba 1968 (Miyawaki 1988, Kolbek et al. 2003); but they are poorly represented on the Badzhal Ridge.

Mountain tundra crowns the column of the vertical vegetation belts. The total cover of vascular plants varies from 30 to $80 \%$. The core of the communities is composed of Ericaceae species: Arctous japonica, Cassiope ericoides, C. tetragona, Ledum decumbens, Phyllodoce caerulea, Rhododendron aureum, R. redowskianum. Usually, the species composition includes also Carex rigidioides, Empetrum sibiricum, Pedicularis labradorica, Saussurea tomentosa, Veratrum anticleoides. The cover of mosses and lichens varies from 20 to $90 \%$; Cetraria islandica, Cladonia arbuscula, C. rangiferina, C. stellaris often occur among other lichens. Aulacomnium turgidum, Hylocomium splendens, Polytrichastrum alpinum, Rhytidium rugosum, Sanionia uncinata and Dicranum spp. are most common among mosses. Tundra communities belong to the class Loiseleurio procumbentis-Vaccinietea Eggler ex Schubert 1960 (syn. 
Cetrario-Loiseleurietea Suz.-Tok. et Umezu 1964) (Miyawaki 1988, Mucina et al. 2016).

Alpine herbaceous communities ("meadows") occur above timberline in local depressions and recesses with continuous winter snowpack that melts off in early summer. Such plots are characterized by a relatively leveled microclimate: in winter they are rather warm due to a snow blanket, and in summer melting snow maintains persistent humidity. Among herbs Aegopodium alpestre, Carex capillaris, Ligularia sibirica, Scorzonera radiata, Sieversia pentapetala, Solidago spiraeifolia, Spiraea beauverdiana are the most frequent. Mosses are mainly associated with rock outcrops and separate boulders; Brachythecium erythrorrhizon, Grimmia hartmanii, Hylocomiastrum pyrenaicum, Lescuraea incurvata, Niphotrichum elongatum, Sanionia uncinata are characteristic for this type of habitat. The syntaxonomy of these communities is unclear.

In addition to the described plant communities, the main types of moss habitats include snow-beds, watercourse banks and beds, cliffs and rock fields (Table 1). Snow-beds are rather rare on the territory due to the relatively low heights of the ridges. Watercourse banks and beds differ in environments and bryophyte composition depending on stream size, altitude, flow rate, substrate, lighting, and so on. Rock outcrops are common on the territory; they occur at all altitudes from river banks to mountain tops, both under forest canopy and in open sites. They house a very diverse composition of mosses, which greatly varies in different belts, between exposed and sheltered sites, with moisture and likely also rock composition. Rock fields and scree also occur throughout an altitudinal range and vary from a few to thousand square meters; if in the highlands, they are caused by weathering processes, otherwise at least part of them are due to the disturbance of vegetation by wildfires. Screes are typically dry and exposed, dominated by lichens with a quite poor and uniform composition of mosses; the most characteristic species are Bucklandiella microcarpa, Cnestrum schistii, Cynodontium asperifolium, Grimmia spp., Hedwigia emodica, Schistidium spp. In the alpine belt, where screes often are moister, such species as Andreaea rupestris, Blindia acuta, Paraleucobryum longifolium, Pogonatum spp., Bucklandiella laeta etc. occur on boulders of screes, and Grimmia becomes more diverse.

\section{I S C USSIO N}

Khabarovsk Territory is one of the insufficiently studied regions of Russia in terms of bryophytes. Only one recently published list of species of Ajan Settlement vicinity and related areas of Dzhukdzhur Mountain Range with 164 moss species (Ignatova et al. 2021) partly uncovers the diversity of mosses of the northern part of the Territory. Southern and middle parts of the Khabarovsk Territory are better explored: relatively complete data is available for the Bureinsky Nature Reserve (Ignatov et al. 2000) and the Botchinsky Nature Reserve (Ignatova et al. 2013) - 283 and 243 taxa correspondingly. There are also preliminary and incomplete lists for the Amur River valley near its mouth (Koponen et al. 1978) - 121 taxa; Bolshoi Khekhtsir (Cherdantseva \& Gambaryan 1986) and Komsomolsk (Cherdantseva \& Gambaryan 1989) Nature Reserves - 156 and 125 taxa; Lanzhinskiye Mountains (Omelko et al. 2010) - 84 taxa; Tardoki-Yani Mountain (Fedosov et al. 2016) - 145 taxa. Additional information is presented in publications about several types of habitats, taxonomic revisions of particular groups, and reports of new findings (the complete bibliography is given in Pisarenko \& Bakalin 2017, Cherdantseva et al. 2018).

Our collections were found to comprise 313 species among, which as many as 73 species are newly recorded for the middle part of Khabarovsk Territory; among the latter, 39 are new to the whole Khabarovsk Territory (Table 1). One species, Campylopus gracilis is first recorded in Russia here. This species was described from Europe, and therefore, the identity of our specimen may be challenged; however, the molecular phylogenetic study by Fedosov et al. (unpublished) indicated its close affinity to European plants of this species. Bryoerythrophyllum chenii was first found in Russia based on specimens from several areas, including Badzhal, Primorye Territory and Jewish Autonomous Region (Kučera et al. 2020). Sematophyllum substrumulosum a predominantly European and Macaronesian species recently found in Transbaikalia (Ignatova et al. 2009) and our record from Badzhal is second for Russia and the whole area outside Europe. Several species were previously assigned in the list with names, which do not fit them well. Actually, several poorly known or undescribed species were revealed within the study area; they will be considered separately within the specially focused taxonomic studies.

The area is hard to penetrate through, and during a month of a field study, we managed to cover only about $25 \mathrm{~km}^{2}$, the resulting list appeared one of the richest in the Russian Far East in the number of species. This is especially interesting bacause the area is not remarkably diverse in terms of landscape, vegetation, and bedrock composition. Moreover, lower altitudes within the area primarily start from $560 \mathrm{~m}$, limiting the representation of "southern" species associated with hemiboreal forests.

A reverse side of managing with a relatively compact but well-studied plot is the lack of confidence why rare species are absent here. In particular, neither Actinothiidium hookerii nor Cryphaea amurensis were found in the studied area. However, both species occur in the upper course of Bureya River (Ignatov et al. 2000) in the ecotopes similar to those surveyed on Badzhal. Indeed, complete surveys hardly exist and in our case, some species may be neglected. This is likely the case of Helodium paludosum, which was not found by us, although it is known from the Badzhal Range based on specimen collected in it northern declivity (MW9075725). On the other hand, according to Ogureeva at al. (2018), the area where occurrences of Actinothuidium and Cryphaea are concentrated in the upper Bureya River, represents not the same biome, as was sampled in Badzhal and these species may be not characteristic for it. This reflects the heterogeneity of Eastern group (see below), among which several species with continental (for instance, Cryphaea amurensis) or predominantly continental (Actinothuidium hookeri) distribution, although likely associated with monsoon circulation occur. Otherwise, the influence of the "third factor" may affect the distribution of this species. 
In recent years, the bryofloras of the Russian Far East several times have been approached by detailed comparative analyses (Pisarenko \& Bakalin 2017, Dudov et al. 2018, Bakalin et al. 2019). In all the cases, analyses showed that the bryofloras of Amur Province, the South part of Khabarovsk Territory and the North of Primorsky Territory are rather close in composition and differ significantly from continental bryofloras located both to the south and to the north and also from insular ones.

So here we limit our comparison by a few floras, which could be considered as more or less completely revealed. The question is the longitudinal gradient itself adequately explains the contribution of Eastern species or sufficient shift of this parameter is associated with a change of biome. For comparison we used (1) the Zeysky Reserve (Dudov et al. 2018) with further additions (Herbarium ... 2021) - 314 species; (2) Upper Bureya (Ignatov et al. 2000) with further additions (Herbarium ... 2021) - 316 species; (3) Botchinsky Nature Reserve (Ignatova et al. 2013) + Tardoki-Yani Mt (Fedosov et al. 2016) with further changes - 303 species (online appendix). The latter two floras were combined since in Botchinsky Reserve range of elevations covers the interval from 230 to $630 \mathrm{~m}$, while in Tordoki-Yani the altitudinal range from 540 to 2090 was sampled, that generally fit that sampled in Badzhal, but with 350 specimens mostly collected in the alpine belt, Tardoki-Yani may only be used in addition to flora where forest belt is well sampled.

The combined list of these four selected bryofloras includes 515 species (online appendix). Only 145 species were recorded in all the bryofloras; another 95 species are registered in some three of them. The intersection of species lists for any pair of compared bryofloras turned out to be at a level of only about $2 / 3$ (Table 2 ). In all cases the number of species noted only in one of the lists, is quite large, ranging from 35 to 49 species (Table 2), that constitutes 11-15\% of the corresponding lists. Even in the considered set of the most fully studied bryofloras the absence of a species in many cases can be attributed to chance. For example, Entodon schleicheri, Helodium blandowii, Hylocomiastrum pyrenaicum, Leptobryum pyriforme, Polytrichum juniperinum, Pseudobryum cinclidioides which are recorded in three lists out of four; Cirriphyllum piliferum, Bryum caespiticium, Drepanocladus aduncus, Entodon concinnus, Leucodon sciuroides, Polytrichum longisetum, Tortella fragilis which are recorded in two lists out of four and so on.

Nevertheless, in many cases, the presence/absence of species can reflect biogeographical trends. It is especially pronounced in the western and northern limits of the "Eastern" species distributions. We considered the group of Eastern species broadly, including species that are predominantly distributed in the eastern sector of cool temperate and boreal zones of Asia or increase their frequency eastwards. Thus, we included in this group amphioceanic species, whose distribution in Asia is caused by the impact of the Pacific Ocean (Oedipodium griffithianum, Sphagnum rubiginosum, Ulota intermedia, etc.), several species, which are also widespread in south Siberia (Anomodon minor, Brothera leana, Claopodium pellucinerve, etc.), or in monsoon climate of Himalayas (Actinothuidium hookeri), but not included central Asian and north Asian species, associated mainly with xeric biomes and/or cryolithozone (Brachythecium boreale, B. jacuticum, Bryoerythrophyllum ferruginascens, Struckia enervis, Lyellia aspera, etc.). About $23 \%$ of moss species revealed within the studied area represent "Eastern element" (online appendix), and this is the most significant proportion of Eastern species among four compared floras. In the Zeysky Reserve and Upper Bureya, this proportion is 14 and $16 \%$, while in the Botchinsky Reserve and Tardoki-Yani, it reaches $20 \%$. At the same time, only 23 eastern species are common between Zeysky Reserve (43 eastern species) and Upper Bureya (50 eastern species). Such difference in the composition of Eastern species may have originated from the rarity of Eastern species on the western limits of their distribution, low ecological amplitude (i.e. association with a narrow range of ecologically suitable ecotopes) and difference in composition of ecotopes between rather dry Zeysky Reserve and more humid Upper Bureya. Badzhal (71 eastern species) have 38 eastern species in common with Upper Bureya and 29 eastern species in common with the Zeysky Reserve, and we suggest that eastward eastern species become more common and thus better represented within studied flora. However, in the Botchinsky Reserve number of Eastern species (60) decreases compared with Badzhal and 44 Eastern species (nearly 3/4) revealed in Botchinsky Reserve and Tardoki-Yani, also occur in Badzhal.

Thirteen of the found on Badzhal eastern species were not noted in other compared bryofloras. Primarly, these are common in the south of Primorye Territory and occur in the southern Kuriles. That are Anomodon solovjovii, Bryoerythrophyllum chenii, Ditrichum macrorbynchum, Entodon sullivantii, Haplohymenium longinerve, Okamuraea hakoniensis, Taxiphyllum aomoriense - the species for which the locations on Badzhal are the most northwestern; and also Dicranum setifolium, Neckera cf. borealis, Niphotrichum elongatum, Plagiothecium japonicum, Rhizomnium tuomikoskii, Sphagnum miyabeanum which are somewhat wider distributed to the west. The reasons for the absence of these species in the Botchinsky Reserve are not entirely clear; perhaps they are not found simply because of their rarity.

For many more species, the locations on the Badzhal are the extreme western or northwestern localities: Arrhenopterum heterostichum, Dilutineuron brevisetum, Echinophyllum sachalinense, Oligotrichum aligerum, O. parallelum, Plagiothecium euryphyllum, Pogonatum contortum, P. japonicum, Pylaisiadelpha tristoviridis, Tetraphis geniculata. For Gollania ruginosa and Eurbynchiadelphus eustegia penetrating the Amur region, the localities on Badzhal mark the northern boundary. For Bartramiopsis lescurii, Bucklandiella laeta, Dicranodontium asperulum, the localities on the Badzhal, along with the Upper Bureya ones mark the western boundary.

In some cases, the fact of the absence of a species is also indicative and clarifies the area boundaries. That refers to several eastern species which are known for Botchinsky Reserve, but so far were found neither in the Amur Region nor in the more western parts of the Khabarovsk Territory (taking into account our efforts on Badzhal): Bryoxiphium japonicum, Climacium japonicum, Oedipodium griffitbianum, Rhytidiadelphus japonicus, Sciuro-bypnum uncinifolium. And same may be suggested for Pleuroziopsis ruthenica with the westernmost locality in the Komsomolsky Reserve (Cherdantseva et al. 2018). 
Since the number of species are of the same level, which is also typical for other montane areas of the southern part of the Russian Far East, we assume that these areas were sampled enough and in all-four curves "number of species/collection effort" largely passed the phase of productive growth; thus way are suitable for comparison. In several runs of cluster analysis with 1000 pseudoreplications of bootstrap different algorithms and distance measures performed in Past 3 for binary lists of species Badzhal invariably formed moderately statistically supported grouping with Botchinsky Reserve + TardokiYani, while topology of remaining terminals differed from analysis to analysis. This result may reflect the impact of biome: in the recent scheme of zoning the territory of Russia by biomes (Ogureeva et al. 2018), the Zeysky Reserve represents Yankano-Dzhagdinsky orobiome, the Bureinsky Reserve - South Ochotian orobiome, while Badzhal Ridge, the Botchinsky Reserve, and the Tordoki-Yani Mountain represent Middle Sikhote-Alinsky variant of the SakhalinSikhote-Alin Orobiome.

Table 2. The intersection matrix for the species lists of the four richest bryofloras of the Amur Region and the Khabarovsk Territory. In brackets is the number of species recorded only in one of the compared bryofloras.

\begin{tabular}{lcccc}
\hline & $\begin{array}{c}\text { Zeysky } \\
\text { Reserve } \\
\mathbf{( 4 9 )}\end{array}$ & $\begin{array}{c}\text { Upper } \\
\text { Bureya } \\
\mathbf{( 4 4 )}\end{array}$ & $\begin{array}{c}\text { Badzhal } \\
\text { range } \\
\mathbf{( 4 0 )}\end{array}$ & $\begin{array}{c}\text { Botchinsky } \\
\text { Reserve \& } \\
\text { Tardoki-Yani } \\
\text { Mt } \\
\mathbf{( 3 5 )}\end{array}$ \\
\hline $\begin{array}{l}\text { Zeysky Reserve } \\
\text { Upper Bureya }\end{array}$ & 314 & 212 & 207 & 203 \\
Badzhal range & & 316 & 214 & 208 \\
$\begin{array}{l}\text { Botchinsky Reserve \& Mt. Tardoki- } \\
\text { Yani }\end{array}$ & & 313 & 219 \\
\hline
\end{tabular}

Table 3. Distribution of moss species of the Zeysky Reserve and the key plot of the Badzhal range by altitude belts, number of species.

\begin{tabular}{llll} 
& \multicolumn{2}{c}{ Species occurence in local floras } \\
\cline { 2 - 4 } Species occurence in vertical belts & $\begin{array}{l}\text { Zeysky } \\
\text { Reserve } \\
\text { only }\end{array}$ & $\begin{array}{l}\text { Common } \\
\text { for both } \\
\text { local } \\
\text { floras }\end{array}$ & $\begin{array}{l}\text { Badzhal } \\
\text { range only }\end{array}$ \\
\hline $\begin{array}{l}\text { Species recorded in the high mountains only } \\
\begin{array}{l}\text { Species recorded both in the forest belt and in the } \\
\text { high mountains }\end{array}\end{array}$ & 11 & 4 & 30 \\
$\begin{array}{l}\text { Species recorded in the forest belt only } \\
\text { Speciny }\end{array}$ & 88 & 97 & 22 \\
\hline
\end{tabular}

Table 4. The inclusion matrix for the species lists by altitude belts of the Zeysky Reserve and the key plot of the Badzhal Mountain Range.

\begin{tabular}{lcccc}
\hline & $\begin{array}{c}\text { Zeysky } \\
\text { Reserve, } \\
\text { forest belt }\end{array}$ & $\begin{array}{c}\text { Zeysky } \\
\text { Reserve, high } \\
\text { mountains }\end{array}$ & $\begin{array}{c}\text { Badzhal } \\
\text { range, forest } \\
\text { belt }\end{array}$ & $\begin{array}{c}\text { Badzhal } \\
\text { range, high } \\
\text { mountains }\end{array}$ \\
\hline $\begin{array}{l}\text { Zeysky Reserve, } \\
\text { forest belt } \\
\begin{array}{l}\text { Zeysky Reserve, } \\
\text { high mountains }\end{array}\end{array}$ & 1 & $\underline{\mathbf{0 . 7 6}}$ & $\underline{\mathbf{0 . 6 7}}$ & 0.57 \\
$\begin{array}{l}\text { Badzhal range, } \\
\text { forest belt }\end{array}$ & 0.2 & 1 & 0.18 & 0.28 \\
$\begin{array}{l}\text { Badzhal range, high } \\
\text { mountains }\end{array}$ & 0.58 & 0.59 & 1 & 0.56 \\
\hline
\end{tabular}

The analysis of species distribution among the three altitudinal vegetation belts of the territory showed that only some species are distributed throughout the whole altitude profile: Amphidium mongeotii, Andreaea rupestris, Aulacomnium turgidum, Bartramia pomiformis, Bartramiopsis lescurii, Blindia acuBucklandiella laeta, Calliergonella lindbergii, Ceratodon purpureus, inm asperifolium, Dicranella curvipes, Dicranum majus, adiceum, Dilutineuron brevisetum, Hylocomium splentrichum elongatum, Oxystegus tenuirostris, Ptilium crista-castrensis, and only about half of them (59 species or $19 \%$ occur solely here. The distribution of species is shown in more detail in the Figure 3.

We also compared species distribution by altitude belts on the Badzhal Ridge and in the Zeysky Reserve, for which such data is available (Dudov et al. 2018, Herbarium ... 2021). About half of the species of the consolidated list of these two territories are common to both bryofloras (Table 3). To ensure the comparability of the data, we have to limit ourselves to distinguishing

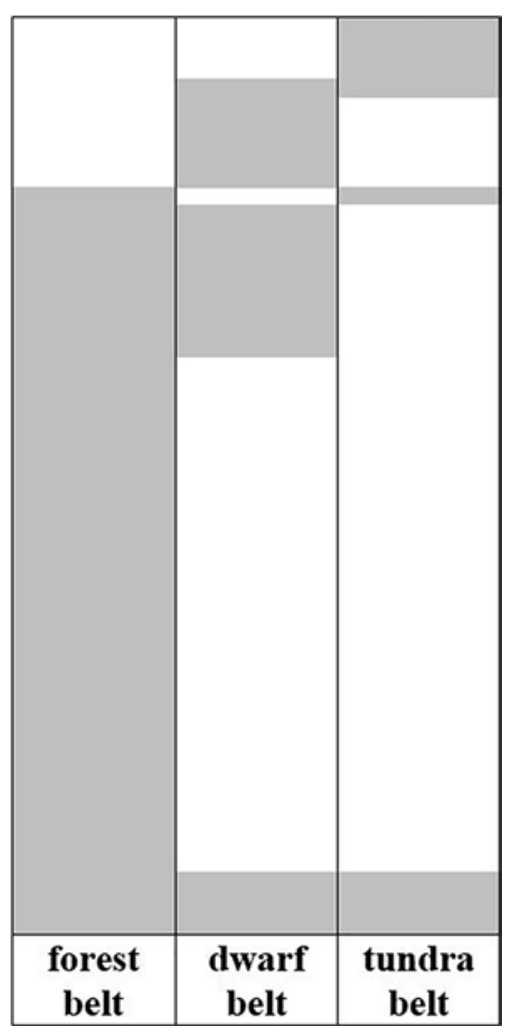

Figure 3 Scheme of quantitative distribution of moss species by altitudinal belts within the studied part of the Badzhal Mountain Range. The height of the gray column shows the number of moss species registered in the corresponding belts 
only two belts: forest belt (comprising "hemiboreal" and "boreal" in case of the Zeysky Reserve) and high mountains (comprising tundra belt and dwarf thickets belt because they were not differentiated for the Zeysky Reserve).

In both areas, most species are associated with the forest belt. The forest belt of the Zeysky Reserve is somewhat more affluent in terms of the number of species. There are several reasons for this. Firstly, in the Zeysky Reserve, the forest belt starts from 315 meters above sea level, whereas on the investigated plot of Badzhal range, the minimum altitude is 522 meters. Hemiboreal, mainly broad-leaved forests growing at 315-500 $\mathrm{m}$ in the Zeya River valley, are enriched by temperate East Asian species, such as Cryphaea amurensis, Drummondia sinensis, Fabronia rostrata, Entodon giraldii, Ptychomitrium sinense, Pylaisia curviramea, Pylaisia falcata; none of them have been found on Badzhal range where such forests absent. The presence of some species in the Zeysky Reserve bryoflora is explained by the large size of the area studied for mosses there and sufficient diversity of ecotopes, covered by bryofloristic studies. So, several species associated mainly with swampy sites (Calliergon giganteum, C. richardsonii, Calliergonella cuspidata, Drepanocladus aduncus, Helodium blandowii, H. paludosum, Sphagnum spp., Warnstorfia fluitans); lime-rich outcrops (Gymnostomum aeruginosum, $\mathrm{Hy}$ menostylium recurvirostrum, Molendoa sendtneriana, Plagiopus oederianus, Seligeria spp., Timmia comata, Timmiella anomala) were found. In the investigated plot of the Badzhal Range, swamps are rare, and limestone outcrops are absent thus, listed species were not found.

Contrary, in the alpine belt more species were recorded in the Badzhal range than in the Zeysky Reserve; in particular because of Arctoa fulvella, A. glacialis, Dicranum angustum, Grimmia funalis, G. hartmanii, G. incurva, G. reflexidens, Lescuraea incurvata, L. saxicola, Oligotrichum aligerum, O. falcatum, Paraleucobryum enerve, Polytrichastrum septentrionale, Pseudoblindia falcata and so on. The trend is rather expected because in the Badzhal Range alpine belt is represented at a larger amplitude of altitudes and occupies larger areas. On the other hand, we expect that actually diversity of alpine ecotopes is low in both areas and alpine bryophyte floras apparently are very close, while the main reason of difference in the number of recorded species is that in Badzhal it was surveyed by bryologists and this starts matter than overall diversity is of the same level.

The belts' species lists inclusion matrix (Table 4) shows low values. That is entirely unexpected, given the vastness of the mosses' areas, compared to which the two territories under consideration are located quite close to each other. We think this differentiation by a relatively sharp climatic and floristic gradient and different species diversity distribution between altitudinal belts. The bryoflora of the Badzhal Mountain Range forest belt is largely included in the bryoflora of the forest belt of the Zeysky Reserve (0.67). The measures of mutual inclusion of the bryoflora of the high mountains are only $0.50-0.57$. Herewith, the bryoflora of the high mountains of the Zeysky Reserve is more included in the bryoflora of the forest belt of it (0.76). However, in the composition of recorded species, the bryoflora of the high mountains of the Badzhal range is more distinctive from the forest belt than the alpine belt in Zeya.The highest diversity of mosses within the study area is associated with rocky habitats: 127 were collected on cliffs, and 38 in rock fields - 142 species in total, and 80 from them occur only in these habitat types, for example Anomodon minor, Grimmia spp., Haplohymenium triste, Herpetineuron toccoae, Struckia enervis, Syntrichia pagorum, Schistidium spp., etc. (Table 1).

Dark coniferous forests in river valley is next habitat in the number of species - 121 species were recorded, of which 50 species are found only here, among them Anomodon thraustus, Callicladium haldaneanum, Pylaisia condensata, $P$. cf. polyantha, Raviella fujisana, Trachycystis ussuriensis and rare Anacamptodon latidens, Arrhenopterum heterostichum, Bryoerythrophyllum chenii, Bryum moravicum, Eurbynchiadelphus eustegia, Homomallium connexum, Hondaella caperata, Plagiomnium confertidens, Pogonatum japonicum, Pylaisiadelpha tristoviridis, Schistostega pennata, Taxiphyllum aomoriense, Tetraphis geniculata are not rare or even common.

Forests on the slopes are poorer in terms of species number: 50 moss species were recorded in Picea and Abies forests and 36 - in Larix forests; examples of species which occur in both types Brothera leana, Dicranum flagellare, D. montanum, Glyphomitrium crispifolium, Heterophyllium nemorosum, Leucodon pendulus, Sciuro-hypnum starkei, Tetraphis pellucida, Ulota rehmannii.

Wetlands are rare in the studied area. We found and examined only a few small massifs, and this explains low diversity of their moss flora - only 34 species among which several species of the genus Sphagnum, Dicranum leioneuron, D. undulatum, Loeskypnum badium, Tomentypnum cf. nitens, Warnstorfia exannulata are most typical and generally associated with bogs. Weak representation of wetlands in the studied area likely resulted in a lack of several groups known as common in boreal and hemiboreal zones, such as Calliergon, and it is hard to imagine Holarctic moss flora without them. 
A large diversity of moss species is associated with watercourse banks and beds that might be due to the heterogeneity of this type; 75 species are recorded here and 40 among them only here: Dichodontium pellucidum, Drepanocladus polygamus, Hygrohypnella ochracea, Philonotis fontana, Plagiomnium ellipticum, Platyhypnum alpestre, Pseudohygrohypnum cf. subeugyrium, Schistidium agassizii, S. platyphyllum, S. sibiricum, Sphagnum beringiense and so on).

The rest types of habitats are poorer in mosses: 16 species are recorded in dwarf pine and dwarf alder thickets and crooked Betula ermanii forests, 13 - in tundra communities, 13 - in alpine "meadows" and 8 - in snow-beds (Table 1).

The largest number of species (88) are recorded on rock surfaces and fissures in rock outcrops; most of them grow only here (Fig. 4). Many species that grow in rocky habitats occupy not rock surfaces but fine earth in cracks, niches and on ledges (62). A lot of species (71) occur on bare humificated soil, 24 from them are noticed only on this kind of substrate, while 19 also grow on plant debris, and 51 species in total were found on plant debris. The next diverse ecological group is pioneers inhabiting eroded mineral ground (40 species). In addition, the group of species inhabiting silty sediments is not large but specific compared with pioneers of eroded mineral ground, among which nearly half inhabited other types of substrates. The epiphytic-epixylic complex is also rather diverse; 82 species were recorded on living trees and logs. Several species can grow on different substrates; in our case 106 taxa, (i.e. ca. $1 / 3$ of the list of species) are not associated with a single type of substrate. Typically these are common and frequent species with wide ecological amplitude.

Regarding the occurrence parameters, among the registered in the key plot mosses 130 species are classified as "rare", and another 69 are classified as "unique". That is, with the total bryophloristic richness of the territory, more than half of the species are threatened with extinction. Obviously, this is due to the anthropogenic transformation of the territory by past fires.

\section{ACKNOWLEDGEMENTS}

The field trip was organized by Pavel Krestov and Vadim Bakalin to whom we are sincerely grateful for an opportunity to take part in it. Field studies were partly supported by RFBR 15-34-20101. Laboratory studies were supported by RSF 1814-00121, and also are the part of biocollection development programs (AAAA-A17-117012610052-2 and USU 440537 for Pisarenko, AAAA-A20-120031990012-4 for Fedosov). We also acknowledge Ministry of Higher Education and Science of Russian Federation for the support the Center of Collective Use "Herbarium MBG RAS", grant 075-15-2021-678.

\section{LITERATURE CITED}

Cherdantseva, V.Ya. \& S.K. Gambaryan 1986. Bryophytes. In: Flora and vegetation of Bolshoi Khekhtsir Nature Reserve (A.B. Melnikova, ed.), pp. 79-101, Vladivostok (in Russian). [Черданцева В.Я., Гамбарян С.К. 1986. Мохообразные // ФАора и растительность Большехехцирского заповедника / под реА. А.Б. Мельниковой. ВАаАивосток. C. 79-101].

Cherdantseva, V.Ya. \& S.K. Gambaryan 1989. Bryophytes. In: Fungi, lichens, algae and bryophytes of Komsomolsk Reserve (Kha- barovsk Territory) (Z.M. Azbukina, V.Ya.Cherdantseva, eds), pp. 109-135, Vladivostok (in Russian). [Черданщева В.Я., Гамбарян С.К. 1989. Мохообразные // Грибы, мишайники, водоросли и мохообразные Комсомольского заповедника (Хабаровский край) / под реА. 3.М. Азбукиной и В.Я. Черданцевой. ВАадивосток. С. 109-135].

Cherdantseva, V.Ya., O.Yu. Pisarenko, M.S. Ignatov, E.A. Ignatova, V.E. Fedosov, S.V. Dudov \& V.A. Bakalin 2018. Mosses of the southern Russian Far East, an annotated check-list. Botanica Pacifica 7(2):53-81.

Cherdantseva, V.Ya., S.K. Gambaryan \& S.V. Osipov 1997. The bryophytes of the upper belts of the Amur-Uda interfluve Mountains (Far East). Botanicheskii Zhurnal 82(1):54-62. (in Russian with English summary). [ЧepАанцева В.Я., Гамбарян С.К., Осипов С.В. 1997. Мохообразные верхних поясов гор Амуро-УАского межАуречья (Аальний Восток) // Ботанический журнал. T. 82, № 1. C. 54-62].

Dokturovskiy, V.S. 1911. Vegetation of the TyrminskoBureinskii district and the Amur Oblast in general. In: Proceedings of the Soil-Botanical Expedition of the study of colonization areas in Russian Asia. Part 2. Botanical studies. Vol. 3, pp. 1-130. St. Petersburg (in Russian). [Аоктуровский B.C. 1911. Растительность Тырминско-Буреинского района и Амурской области вообще // Труды почвенноботанической экспедиции по исследованию колонизационных районов азиатской России. Ч. II. Ботанические исследования. Вып. 3. СПб. 130 с.].

Dudov, S.V., M.N. Kozhin, V.E. Fedosov \& E.A. Ignatova 2018. Moss flora of Zeysky State Nature Reserve (Tukuringra Range, Amur Province, Russia). Botanica Pacifica 7(2):83-104.

Ellis, L.T., M. Aleffi, H. Bednarek-Ochyra, V.A. Bakalin, M. Boiko, J.A. Calleja, V.E. Fedosov, M.S. Ignatov, E.A. Ignatova, R. Garilleti, T. Hallingbäck, N. Lönnell, N. Hodgetts, T. Kiebacher, J. Larraín, M. Lebouvier, M. Lüth, V. Mazimpaka, B. Vigalondo, F. Lara, R. Natcheva, M. Nobis, A. Nowak, J.D. Orgaz, J. Guerra, J. Pantović, N. Nikolić, M.S. Sabovljević, A.D. Sabovljević, O.Yu. Pisarenko, V. Plášek, Z. Skoupá, S. Poponessi, M. Privitera, M. Puglisi, M. Skudnik \& Q.H. Wang 2017. New national and regional bryophyte records, 51. Journal of Bryology 39(2):177-190.

Ellis, L.T., O.M. Afonina, R.L. Andriamiarisoa, G. Asthana, R. Bharti, P. Aymerich, B. Bambe, M. Boiko, M. Brugués, E. Ruiz, L. Sáez, M.J. Cano, R. Ros, L. C`ihal, J. Deme, J. Csiky, G. Dihoru, P. Dřevojan, T. Ezer, V.E. Fedosov, E.A. Ignatova, A.P. Seregin, C.A. Garcia, A. Martins, C. Sérgio, M. Sim-Sim, A.S.B. Rodrigues, S.R. Gradstein, C. Reeb, A. Irmah, M. Suleiman, T. Koponen, J. Kučera, M. Lebouvier, Y. LiQun, D.G. Long, A.I. Maksimov, T.A. Maksimova, J. Muñoz, M. Nobis, A. Nowak, R. Ochyra, S.V. O’Leary, F. Osorio, O.Yu. Pisarenko, V. Plášek, Z. Skoupá, A. SchäferVerwimp, N. Schnyder, J.R. Shevock, S. Ștefănut, M. Sulayman, B.-Y. Sun, S.J. Park, D.Ya. Tubanova, † J. Vánă, G.J. Wolski, K.-Y. Yao, Y-J Yoon \& E. Yücel 2018. New national and regional bryophyte records, 56. Journal of Bryology 40(3):271-296.

Fedosov V.E., A.V. Ermolenko, D.E. Koltysheva \& V.A. Bakalin 2016. On the moss flora of Tardoki-Yani Mountain (North Sikhote-Alin, Russian Far East). Arctoa 25(2):420-428.

Gozhev, A.D. Forests of the Udskii district. In: Amgun'Selemdrhinsk expedition of the USSR Academy of Sciences. Part 2. Udsk-Selemdzhinsk detachment (V.L. Komarov, ed.), pp. 53-106, Izdatel'stvo akademii nauk SSSR, Leningrad (in Russian). [Гожев А.А. 1934. Аеса УАского района // Амгунь-Селемджинская экспедиция Академии наук СССР, ч. 2. УАско-СелемАжинский отряА / поА реА В.А. Комарова. Аенинград: АН СССР. С. 53-106]. 
Herbarium specimens of Russian mosses. 2021. Available from: http://arctoa.ru/Flora/basa.php Last accessed 17.11.2021

Ignatov, M.S., B.C. Tan, Z. Iwatsuki \& E.A. Ignatova 2000. Moss flora of the Upper Bureya River (Russian Far East). Journal of the Hattori Botanical Laboratory 88:147-178.

Ignatov, M.S., E.A. Ignatova, V.E. Fedosov, E.I. Ivanova, H.H. Blom, J. Muñoz, H. Bednarek-Ochyra, O.M. Afonina, L.E. Kurbatova, I.V. Czernyadjeva \& V.Ya. Cherdantseva 2017. Moss flora of Russia. Vol. 2. Oedipodiales-Grimmiales (M.S. Ignatov, ed.). KMK Scientific Press Ltd., Moscow, 560 pp. (in Russian and English). Игнатов M.С., Игнатова E.A., Федосов В.Э., Иванова Е.И., Блом Х.Х., Муньос И., БеАнарек-Охыра X., Афонина O.M., Курбатова А.Е., Чернядьева И.В., Черданцева В.Я. 2017. ФАора мхов России. Tом 2. Oedipodiales - Grimmiales. Москва: КМК. 560 с.].

Ignatova, E.A., B.C. Tan, O.M. Afonina \& M.S. Ignatov 2009. Sematophyllum (Sematophyllaceae, Bryophyta), a new genus and family for Russia. Arctoa 18:213-216.

Ignatova, E.A., M.S. Ignatov, K.G. Klimova \& V.A. Bakalin 2021. Contribution to the moss flora of Ayan (west coast of the Sea of Okhotsk, the Russian Far East). Arctoa 30(1):79-90.

Ignatova, E.A., V.Ya. Cherdantseva, O.V. Ivanov, I.V. Kostomarova \& M.S. Ignatov 2013. A preliminary list of mosses of the Botchinsky State Nature Reserve (Russian Far East). Arctoa 22(1):207-216.

Ivanov, O.V., M.A. Kolesnikova, T.V. Akatova, O.M. Afonina, E.Z. Baisheva, A.G. Bezgodov, O.A. Belkina, I.V. Czernyadjeva, S.V. Dudov, V.E. Fedosov, E.I. Ivanova, E.A. Ignatova, M.N. Kozhin, E.D. Lapshina, A.A. Notov, O.Yu. Pisarenko, N.N. Popova, A.N. Savchenko, V.V. Teleganova, D.Ya. Ukrainskaya \& M.S. Ignatov 2017. The database of the moss flora of Russia. Arctoa 26(1):1-10.

Kolbek, J., M. Valachovic, N. Ermakov \& Z. Neuhauslova 2003. Comparison of forest syntaxa and types in Northeast Asia. In: Forest vegetation of Northeast Asia (J. Kolbek, M. Šrůtek, E. Box., eds), pp. 409-423, Kluwer Academic Publishers, Dordrecht.

Koponen, T., I. Järvinen \& P. Isoviita 1978. Bryophytes from the Soviet Far East, mainly the Khabarovsk territory. Annales Botanici Fennici 15:107-121.

Krestov, P.V. \& Y. Nakamura. 2002. Phytosociological study of the Picea jezoensis forests of the Far East. Folia Geobotanica 37(4):441-474.

Krestov, P.V. 2003. Forest vegetation of easternmost Russia (Russian Far East). In: Forest Vegetation of Northeast Asia (J. Kolbek, M. Šrůtek, E.O. Box, eds), pp. 93-180, Kluwer Academic Publishers, Dordrecht.

Krestov, P.V., N.B. Ermakov, S.V. Osipov \& Y. Nakamura 2009. Classification and phytogeography of larch forests of Northeast Asia. Folia Geobotanica 44(4):323-363.

Kučera, J., P. Sollman, O.M. Afonina, E.A. Ignatova, V.E. Fedosov, J.R. Shevock, D.Ya. Tubanova \& M.S. Ignatov 2020. Range extensions for Bryoerythrophyllum sollmanianum and Tortula yuennanensis (Pottiaceae, Musci) with reconsideration of their phylogenetic affinities including Pararbexophyllum, gen. nov. Nova Hedwigia 150:273-292.

Manko, Yu.I. 1961. The feature article about forest vegetation of upper half of Urmi River Basin. V.L. Komarov Memorial Lectures 9:42-71 (in Russian). [Манько Ю.И. 1961. Краткий очерк месной растительности верхней половины бассейна реки Урми // Комаровские чтения. Т. 9. С. 42-71].

Manko, Yu.I. \& V.A. Rosenberg 1967. An outline of vegetation of the Amur-Amgun interfluve. Vegetation of the northern regions of the Far East. V.L. Komarov Memorial Lectures 14:59-85 (in Russian). [Манько Ю.И., Розенберг В.А. 1967. Очерк растительности межАуречья Амур-Амгунь. Растительность северных районов Аальнего Востока // Комаровские чтения. Т. 14. С. 59-85].

Miyawaki, A. (ed.) 1988. Vegetation of Japan. Vol. 9. Hokkaido. Shibundo, Tokyo, 563 pp. (in Japanese with German and English synopses).

Mucina, L., H. Bültmann, K. Dierßen, J.-P. Theurillat, T. Raus, A. Čarni, K. Šumberová, , W. Willner, J. Dengler, R.G. García, M. Chytrý, M. Hájek, R. Di Pietro, D. Iakushenko, J. Pallas, F.J. Daniëls, E. Bergmeier, A. Santos Guerra, N. Ermakov, M. Valachovič, J.H. Schaminée, T. Lysenko, Y.P. Didukh, S. Pignatti, J.S. Rodwell, J. Capelo, H.E. Weber, A. Solomeshch, P. Dimopoulos, C. Aguiar, S.M. Hennekens \& L. Tichý 2016. Vegetation of Europe: hierarchical floristic classification system of vascular plant, bryophyte, lichen, and algal communities. Applied Vegetation Science 19:3-264.

Nakamura, Y., P.V. Krestov \& A.M. Omelko. 2007. Bioclimate and zonal vegetation in Northeast Asia: first approximation to an integrated study. Phytocoenologia 37(3-4):443-470.

Ogureeva, G.N., N.B. Leonova, E.V. Buldakova, N.G. Kadetov, M.V. Arkhipova, I.M. Miklyaeva, M.V. Bocharnikov, S.V. Dudov, E.A. Ignatova, M.S. Ignatov, E.E. Muchnik, G.P. Urbanavichus, V.Yu. Rumyantsev, A.K. Danilenko, L.G. Emelyanova, O.A. Leonteva, A.A. Romanov \& P.A. Konstantinov 2018. The biomes of Russia. Map. Scale 1:750000, Second revised eddition (in Russian). [Огуреева Г.Н., Аеонова Н.Б., Булдакова Е.В., Кадетов Н.Г., Архипова М.В., Микляева И.М., Бочарников М.В., Аудов С.В., Игнатова Е.А., Игнатов М.С., Мучник Е.Э., Урбанавичюс Г.П., Ааниленко А.К., Румянцев В.Ю., Емельянова А.Г., Аеонтьева О.А., Романов А.А., Константинов П.А. 2018. Биомы России. Карта. Масштаб 1:7 500 000]. Available from: https://wwf.ru/what-wedo/bio/biomy-rossii/. Last accessed 17.11.2021.

Omelko, A.M., V.V. Yakubov, V.A. Bakalin, A.V. Velikanov, V.Ya. Cherdantseva, I.F. Skirina, A.N. Yakovleva \& P.V. Krestov 2010. Plant cover of the Mountains Lanzhinskiye Gory (Okhotia). V.L. Komarov Memorial Lectures 57:103-163 (in Russian with English summary). [Омелько А.М., Якубов В.В., Бакалин В.А., Великанов А.В., Черданцева В.Я., Скирина И.Ф., Яковлева А.Н., Крестов П.В. 2010. РаститеАьный покров Аанжинских гор (Охотия) // Комаровские чтения. Т. 57. С. 103-163].

Orlov, A.Ya. 1955. Coniferous forests of the Amgun and Bureya interfluve area. Izdatelstvo AN SSSR, Moscow, 208 pp. (in Russian). [Орлов А.Я., 1955. Хвойные леса Амгунь-Буреинского межАуречья. М.: ИзА-во. АН СССР. 208 с.].

Osipov, S.V. 2002. Vegetation cover of taiga-golets landscapes of the Bureinskiy highland. Dalnauka, Vladivostok, 378 pp. (in Russian). [Осипов C.В. 2002. Растительный покров таежно-гольцовых мандшафтов Буреинского нагорья. ВАадивосток: АаАьнаука. 378 с.].

Petrov, E.S., P.V. Novoritskiy, V.T. Lenshin. 2000. Climate of the Khabarovsk Territory and the Jewish Autonomous Region. Dalnauka, Vladivostok, Khabarovsk, 170 pp. (in Russian). [Петров Е.С., Новороцкий П.В., Аеншин В.Т. 2000. Климат Хабаровского края и Еврейской автономной области. ВАаАивосток; Хабаровск: Аальнаука. 174 с.].

Pisarenko O.Yu., Fedosov V.E., Bakalin V.A. Mosses of the Russian Far East Exiccatae. Fasc.III (№ № 71-105). Vladivostok, 2017. 14 pp.

Pisarenko, O.Yu. \& V.A. Bakalin 2017. Moss Diversity Distribution Patterns and floral agglomerates in the 
Russian Far East. Botanica Pacifica. A journal of plant science and conservation. 6(2):21-33.

Pisarenko, O.Yu. \& V.E. Fedosov 2016. New moss records from Khabarovsk Territory. 3. New bryophyte records. 7 (E.V. Sofronova, ed.). Arctoa 25:446-447.

Rosenberg, V.A. 1959. Dark coniferous forests of the northern end of the Sikhote-Alin. Soobshcheniya DV F SO AN SSSR 2:17-23, Vladivostok. (in Russian). [Розенберг В.А. 1959. Темнохвойные меса северной оконечности Сихотэ-А^иня / / Сообщения АВФ СО АН СССР. Вып. 2. ВАаАивосток. С. 17-23].

Shlotgauer, S.D. 1990. Plant world of the suboceanic highlands. Nauka, Moscow, 224 pp. (in Russian). ШАотгауэр C.А. 1990. Растительный мир субокеанических высокогорий. М: Наука. 224 с.].

Shlotgauer, S.D. 2014. Specifics of development of highmountain flora in the Badzhal Range (Khabarovsk kray). Contemporary Problems of Ecology 1:26-31.

Shlotgauer, S.D. 2015. Vascular plants of the southwestern part of the Badzhal Ridge (Khabarovsk region). Botanicheskii Zhurnal 100(7):697-709 (in Russian with English summary). ШАотгауэр С.А. 2015. Сосудистые растения
юго-запаАной части БаАжальского хребта (Хабаровский край) // Ботанический журнац Т. 100, № 7. C. 697-709].

Sochava, V.B. 1934. Vegetation cover of the Bureinskiy Mountain Range to the north of the Dul'nilanskiy Pass. In: Proceedings of the Council for the Study of Productive Forces. Far Eastern Series. Vol. 2. The Amgun'-Selemdja Expedition. Part 1. Bureya Party (V.L. Komarov, ed.), pp. 109-242, Izdatelstvo AN SSR, Leningrad (in Russian). [Сочава В.Б. 1934. Растительный покров Буреинского хребта к северу от Аульниканского перевала // Труды совета по изучению производительных сил. Серия Аальневосточная, вып. 2. Амгунь-СемемАжинская экспедиция Академии наук СССР [Текст] : научное издание. Ч. 1. Буреинский отряд / реА. В.А. Комаров. $\Lambda .:$ ИзА-во АН CСCP. C. 109-242].

Sochava, V.B. 1956. Larch forests. In: Vegetation cover of USSR. Explanatory text for Geobotanical map of USSR, part 1. Izd-vo AN SSSR, Moscow-Leningrad (in Russian). [Сочава В.Б. I956. Аиственничные ^еса // Растительный покров СССР. Пояснительный текст к Геоботанической карте СССР, ч. I. М.-А.: ИзА-во АН СССР.]. 